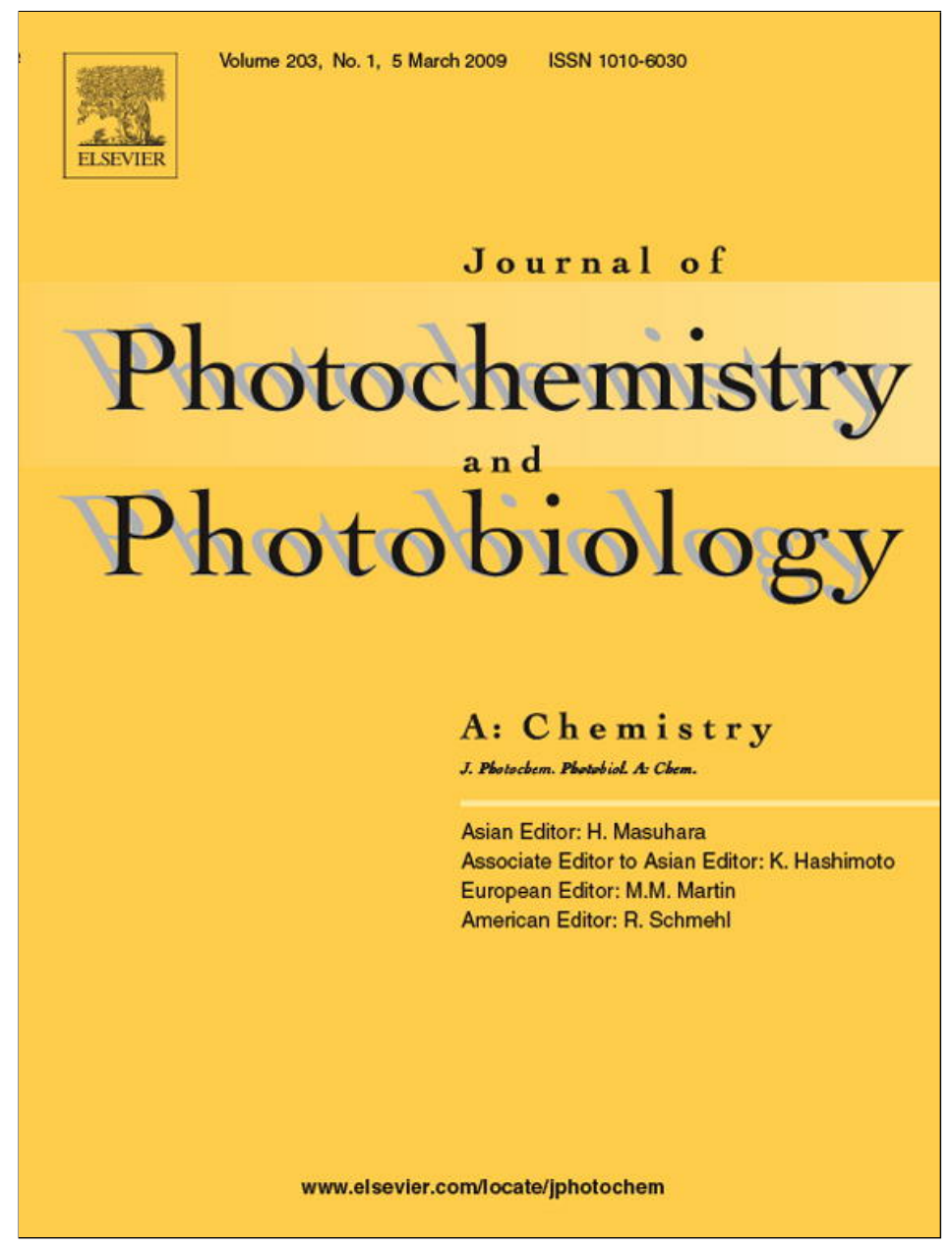

This article appeared in a journal published by Elsevier. The attached copy is furnished to the author for internal non-commercial research and education use, including for instruction at the authors institution and sharing with colleagues.

Other uses, including reproduction and distribution, or selling or licensing copies, or posting to personal, institutional or third party websites are prohibited.

In most cases authors are permitted to post their version of the article (e.g. in Word or Tex form) to their personal website or institutional repository. Authors requiring further information regarding Elsevier's archiving and manuscript policies are encouraged to visit:

http://www.elsevier.com/copyright 


\title{
Synthesis and properties of novel chemiluminescent biological probes: 2- and 3-(2-Succinimidyloxycarbonylethyl)phenyl acridinium esters
}

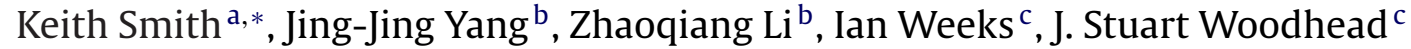 \\ a School of Chemistry, Cardiff University, Main Building, Park Place, Cardiff, CF10 3AT, UK \\ ${ }^{\mathrm{b}}$ Centre for Clean Chemistry, Department of Chemistry, University of Wales Swansea, Swansea, SA2 8PP, UK \\ ${ }^{c}$ Molecular Light Technology Research Limited, 5 Chiltern Close, Cardiff Industrial Park, Cardiff, CF4 5DL, UK
}

\section{A R T I C L E I N F O}

\section{Article history:}

Received 10 June 2008

Received in revised form 12 December 2008

Accepted 17 December 2008

Available online 25 December 2008

\section{Keywords:}

Acridinium ester

Chemiluminescence

Chemiluminescence immunoassay

\begin{abstract}
A B S T R A C T
Five new chemiluminescent aryl acridiniumcarboxylate esters 6-10, with a linker group in an orthoor meta-position of the phenoxy ring, have been synthesized. The ortho- derivatives $\mathbf{6}, \mathbf{7}$ and 9 show significant improvement in the quantum yield of chemiluminescence and slower chemiluminescence kinetics compared to the unsubstituted para- derivative, 1, while the ortho- derivative $\mathbf{8}$ shows quicker chemiluminescence kinetics and a lower quantum yield of chemiluminescence. The meta- derivative $\mathbf{1 0}$ shows similar chemiluminescent properties to that of its para-substituted analogue.
\end{abstract}

(C) 2009 Elsevier B.V. All rights reserved.

\section{Introduction}

Chemiluminescent acridinium esters are widely used as labels in biochemical binding assays such as immunoassay [1] and nucleic acid hybridisation assays [2]. Accordingly, it is desirable to understand the structural features that influence the parameters of the chemiluminescent reaction, such as kinetics and intensity, which will ultimately govern the utility of this class of molecules as labels. In particular, it is desirable to be able to quantify the chemiluminescence emission both rapidly and with a high signal-background ratio to enable convenient and high sensitivity assays to be developed. In a preceding paper [3], we discussed the synthesis and application of an initial series of acridinium esters (Scheme 1, compounds 2-5) that differed from an established acridinium ester (compound 1) in having substituents in the ortho-position of the phenyl group. It would be of interest to know whether placement of the linker group at the ortho- or meta- rather than the para-position of the phenyl ring would influence the chemiluminescent reaction. As a continuation of our previous work, we report here the synthesis and chemiluminescent properties of a second series of acridinium esters (Scheme 1, compounds 6-10). This study should aid understanding of the factors influencing acridinium ester chemiluminescence and ultimately permit the design of labels having improved properties, relative to those labels described todate, for any given application.

\footnotetext{
* Corresponding author. Tel.: +44 2920-870600; fax: +44 2920870600 .

E-mail address: smithk13@cardiff.ac.uk (K. Smith).
}

\section{Experimental}

\subsection{General methods}

Melting points (mp) were recorded on a Griffin Melting Point Apparatus and are reported uncorrected. IR spectra were obtained using a Perkin Elmer FT-IR spectrometer $1725 \times .{ }^{1} \mathrm{H}$ NMR $(400 \mathrm{MHz})$ and ${ }^{13} \mathrm{C}$ NMR $(100 \mathrm{MHz})$ spectra were measured on a Bruker AC 400 spectrometer with tetramethylsilane as internal standard. Chemical shifts $(\delta)$ are expressed in ppm, and coupling constants $(J)$ are in $\mathrm{Hz}$. Mass spectra were recorded on a VG 12/253J mass spectrometer for low-resolution electron impact (EI) and chemical ionisation (CI) measurements and a VG AutoSpec mass spectrometer for fast atom bombardment (FAB) mass spectra. The data are presented as $\mathrm{m} / \mathrm{z}$ ratios for the molecular ion and several of the most abundant other ions with their percent relative intensities given in brackets. Microanalyses were obtained from a Carlo Erba 1106 instrument by the microanalysis laboratory at Cardiff University. Column chromatography was carried out with silica gel 60 (230-400 mesh, Merck). Chromatotron separation was carried out on chromatotron Model 7924T from TC Research using $4 \mathrm{~mm}, 2 \mathrm{~mm}$ or $1 \mathrm{~mm}$ thick silica gel $60 \mathrm{GF}_{258}$ (Merck) layers. In order to help in the assignments, expected chemical shifts were calculated using additivity values and model compounds. [4] Assignments of signals having similar chemical shifts and coupling patterns have not been rigorously confirmed.

\subsection{Syntheses}

The synthetic routes are given in Schemes 2 and 3. 
<smiles>[R2]c1cc(CCC(=O)ON2C(=O)CCC2=O)cc([R])c1OC(=O)c1c2ccccc2[n+](C)c2ccccc12</smiles><smiles>[R2]c1cc([R])c(OC(=O)c2c3ccccc3[n+](C)c3ccccc23)c(CCC(=O)OC2C(=O)CCC2=O)c1</smiles><smiles>C[n+]1c2ccccc2c(C(=O)Oc2cccc(CCC(=O)ON3C(=O)CCC3=O)c2)c2ccccc21</smiles>
1. $\mathrm{R}_{1}=\mathrm{R}_{2}=\mathrm{H}$
2. $\mathrm{R}_{1}=\mathrm{R}_{2}=\mathrm{CH}_{3}$
3. $\mathrm{R}_{1}=\mathrm{R}_{2}=\mathrm{OCH}_{3}$
4. $\mathrm{R}_{1}=\mathrm{H}, \mathrm{R}_{2}=\mathrm{OCH}_{3}$
5. $\mathrm{R}_{1}=\mathrm{R}_{2}=\mathrm{Br}$

6. $\mathrm{R}_{1}=\mathrm{R}_{2}=\mathrm{H}$

7. $\mathrm{R}_{1}=\mathrm{R}_{2}=\mathrm{CH}_{3}$

8. $\mathrm{R}_{1}=\mathrm{R}_{2}=\mathrm{Br}$

9. $\mathrm{R}_{1}=\mathrm{CH}_{3}, \mathrm{R}_{2}=\mathrm{Br}$

10.

Scheme 1. Acridinium esters 1-10.<smiles>[R]c1cc([R])c(O)c([R])c1[2H]</smiles>

6a: $\mathrm{R}^{1}=\mathrm{R}^{2}=\mathrm{R}^{3}=\mathrm{H}, \mathrm{R}^{4}=\mathrm{CH}_{2} \mathrm{CH}_{2} \mathrm{CO}_{2} \mathrm{H}$

7a: $\mathrm{R}^{1}=\mathrm{R}^{2}=\mathrm{CH}_{3}, \mathrm{R}^{3}=\mathrm{H}, \mathrm{R}^{4}=\mathrm{CH}_{2} \mathrm{CH}_{2} \mathrm{CO}_{2} \mathrm{H}$ $8 \mathrm{a}: \mathrm{R}^{1}=\mathrm{R}^{2}=\mathrm{Br}, \mathrm{R}^{3}=\mathrm{H}, \mathrm{R}^{4}=\mathrm{CH}_{2} \mathrm{CH}_{2} \mathrm{CO}_{2} \mathrm{H}$ 9a: $\mathrm{R}^{1}=\mathrm{CH}_{3}, \mathrm{R}^{2}=\mathrm{Br}, \mathrm{R}^{3}=\mathrm{H}, \mathrm{R}^{4}=\mathrm{CH}_{2} \mathrm{CH}_{2} \mathrm{CO}_{2} \mathrm{H}$ 10a: $R^{1}=R^{2}=H, R^{3}=\mathrm{CH}_{2} \mathrm{CH}_{2} \mathrm{CO}_{2} \mathrm{H}, \mathrm{R}^{4}=\mathrm{H}$<smiles>[R]c1cc([R])c(O)c([R7])c1[R]</smiles>

6b: $R^{1}=\mathrm{R}^{2}=\mathrm{R}^{5}=\mathrm{H}, \mathrm{R}^{6}=\mathrm{CH}_{2} \mathrm{CH}_{2} \mathrm{CO}_{2} \mathrm{CH}_{2} \mathrm{Ph}(90 \%)$ 7b: $\mathrm{R}^{1}=\mathrm{R}^{2}=\mathrm{CH}_{3}, \mathrm{R}^{5}=\mathrm{H}, \mathrm{R}^{6}=\mathrm{CH}_{2} \mathrm{CH}_{2} \mathrm{CO}_{2} \mathrm{CH}_{2} \mathrm{Ph}(81 \%)$ 8b: $R^{1}=R^{2}=\mathrm{Br}, \mathrm{R}^{5}=\mathrm{H}, \mathrm{R}^{6}=\mathrm{CH}_{2} \mathrm{CH}_{2} \mathrm{CO}_{2} \mathrm{CH}_{2} \mathrm{Ph}(88 \%)$ 9b: $\mathrm{R}^{1}=\mathrm{CH}_{3}, \mathrm{R}^{2}=\mathrm{Br}, \mathrm{R}^{5}=\mathrm{H}, \mathrm{R}^{6}=\mathrm{CH}_{2} \mathrm{CH}_{2} \mathrm{CO}_{2} \mathrm{CH}_{2} \mathrm{Ph}(70 \%)$ 10b: $\mathrm{R}^{1}=\mathrm{R}^{2}=\mathrm{H}, \mathrm{R}^{5}=\mathrm{CH}_{2} \mathrm{CH}_{2} \mathrm{CO}_{2} \mathrm{CH}_{2} \mathrm{Ph}, \mathrm{R}^{6}=\mathrm{H}(84 \%)$

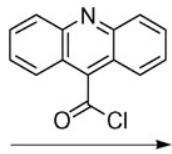<smiles>[R]c1cc([R])c(OC(=O)c2c3ccccc3nc3ccccc23)c([R])c1[R7]</smiles>

6c: $\mathrm{R}^{1}=\mathrm{R}^{2}=\mathrm{R}^{5}=\mathrm{H}, \mathrm{R}^{6}=\mathrm{CH}_{2} \mathrm{CH}_{2} \mathrm{CO}_{2} \mathrm{CH}_{2} \mathrm{Ph}(85 \%)$ $7 \mathrm{c}: \mathrm{R}^{1}=\mathrm{R}^{2}=\mathrm{CH}_{3}, \mathrm{R}^{5}=\mathrm{H}, \mathrm{R}^{6}=\mathrm{CH}_{2} \mathrm{CH}_{2} \mathrm{CO}_{2} \mathrm{CH}_{2} \mathrm{Ph}(70 \%)$ 8c: $R^{1}=R^{2}=\mathrm{Br}, \mathrm{R}^{5}=\mathrm{H}, \mathrm{R}^{6}=\mathrm{CH}_{2} \mathrm{CH}_{2} \mathrm{CO}_{2} \mathrm{CH}_{2} \mathrm{Ph}(85 \%)$ 9c: $\mathrm{R}^{1}=\mathrm{CH}_{3}, \mathrm{R}^{2}=\mathrm{Br}, \mathrm{R}^{5}=\mathrm{H}, \mathrm{R}^{6}=\mathrm{CH}_{2} \mathrm{CH}_{2} \mathrm{CO}_{2} \mathrm{CH}_{2} \mathrm{Ph}(75 \%)$ 10c: $R^{1}=R^{2}=H, R^{5}=C_{2} C_{2} C_{2} C_{2} \mathrm{CH}_{2} P h, R^{6}=H(56 \%)$<smiles>[R]c1cc([R])c(OC(=O)c2c3ccccc3[n+](C)c3ccccc23)c([R])c1[R]</smiles>

6: $\mathrm{R}^{1}=\mathrm{R}^{2}=\mathrm{R}^{7}=\mathrm{H}, \mathrm{R}^{8}=\mathrm{CH}_{2} \mathrm{CH}_{2} \mathrm{CONHS}^{*}(92 \%)$ 7: $R^{1}=R^{2}=\mathrm{CH}_{3}, R^{7}=\mathrm{H}, \mathrm{R}^{8}=\mathrm{CH}_{2} \mathrm{CH}_{2} \mathrm{CONHS}^{*}(90 \%)$ 8: $R^{1}=R^{2}=\mathrm{Br}, \mathrm{R}^{7}=\mathrm{H}, \mathrm{R}^{8}=\mathrm{CH}_{2} \mathrm{CH}_{2} \mathrm{CONHS}^{*}(86 \%)$ 9: $\mathrm{R}^{1}=\mathrm{CH}_{3}, \mathrm{R}^{2}=\mathrm{Br}, \mathrm{R}^{7}=\mathrm{H}, \mathrm{R}^{8}=\mathrm{CH}_{2} \mathrm{CH}_{2} \mathrm{CONHS}^{*}(82 \%)$ 10: $R^{1}=R^{2}=H, R^{7}=C_{2} C_{2} C_{2} C_{N H S}^{*}, R^{8}=H(71 \%)$<smiles>[R]c1cc([R])c(OC(=O)c2c3ccccc3nc3ccccc23)c([R])c1[R]</smiles>

6e: $\mathrm{R}^{1}=\mathrm{R}^{2}=\mathrm{R}^{7}=\mathrm{H}, \mathrm{R}^{8}=\mathrm{CH}_{2} \mathrm{CH}_{2} \mathrm{CONHS}^{*}(80 \%)$ 7e: $R^{1}=R^{2}=\mathrm{CH}_{3}, R^{7}=\mathrm{H}, \mathrm{R}^{8}=\mathrm{CH}_{2} \mathrm{CH}_{2} \mathrm{CONHS}^{*}(90 \%)$ $8 \mathrm{e}: \mathrm{R}^{1}=\mathrm{R}^{2}=\mathrm{Br}, \mathrm{R}^{7}=\mathrm{H}, \mathrm{R}^{8}=\mathrm{CH}_{2} \mathrm{CH}_{2} \mathrm{CONHS}^{*}(75 \%)$ 9e: $\mathrm{R}^{1}=\mathrm{CH}_{3}, \mathrm{R}^{2}=\mathrm{Br}, \mathrm{R}^{7}=\mathrm{H}, \mathrm{R}^{8}=\mathrm{CH}_{2} \mathrm{CH}_{2} \mathrm{CONHS}^{*}(70 \%)$ 10e: $R^{1}=R^{2}=H, R^{7}=C_{2} C_{2} C_{2 O N H S}{ }^{*}, R^{8}=H(67 \%)$<smiles>[R]c1cc([R])c(OC(=O)c2c3ccccc3nc3ccccc23)c([R])c1[R]</smiles>

6d: $\mathrm{R}^{1}=\mathrm{R}^{2}=\mathrm{R}^{3}=\mathrm{H}, \mathrm{R}^{4}=\mathrm{CH}_{2} \mathrm{CH}_{2} \mathrm{CO}_{2} \mathrm{H}(90 \%)$ $7 d: R^{1}=R^{2}=\mathrm{CH}_{3}, R^{3}=\mathrm{H}, \mathrm{R}^{4}=\mathrm{CH}_{2} \mathrm{CH}_{2} \mathrm{CO}_{2} \mathrm{H}(80 \%)$ 8d: $\mathrm{R}^{1}=\mathrm{R}^{2}=\mathrm{Br}, \mathrm{R}^{3}=\mathrm{H}, \mathrm{R}^{4}=\mathrm{CH}_{2} \mathrm{CH}_{2} \mathrm{CO}_{2} \mathrm{H}(80 \%)$ 9d: $\mathrm{R}^{1}=\mathrm{CH}_{3}, \mathrm{R}^{2}=\mathrm{Br}, \mathrm{R}^{3}=\mathrm{H}, \mathrm{R}^{4}=\mathrm{CH}_{2} \mathrm{CH}_{2} \mathrm{CO}_{2} \mathrm{H}(85 \%)$ 10d: $R^{1}=R^{2}=H, R^{3}=\mathrm{CH}_{2} \mathrm{CH}_{2} \mathrm{CO}_{2} \mathrm{H}, \mathrm{R}^{4}=\mathrm{H}(78 \%)$

Reagents: (i) $\mathrm{PhCH}_{2} \mathrm{OH},\left(\mathrm{CF}_{3} \mathrm{CO}\right.$ ) $)$; (ii) $\mathrm{HBr}, \mathrm{CH}_{3} \mathrm{CO}_{2} \mathrm{H}$; (iii) $\mathrm{N}$-hydroxysuccinimide, dicyclohexylcarbodiimide; (iv) $\mathrm{CF}_{3} \mathrm{SO}_{3} \mathrm{Me}$; (v) $\mathrm{PhCH} 2 \mathrm{Cl}, \mathrm{KOH}$

${ }^{\star} \mathrm{NHS}=$<smiles>C[14CH2][14CH2]ON1C(=O)CCC1=O</smiles>

Scheme 2. General strategy for the synthesis of acridinium ester labels. 
<smiles>Cc1ccccc1CCC(=O)O</smiles><smiles>Cc1ccc(O)c(C)c1</smiles>

$7 \mathbf{f}$<smiles>Cc1cc(C)c2c(c1)CCC(=O)O2</smiles>

$7 \mathrm{~g}$<smiles>Cc1cc(C)c(O)c(CCC(=O)O)c1</smiles>

$7 a$

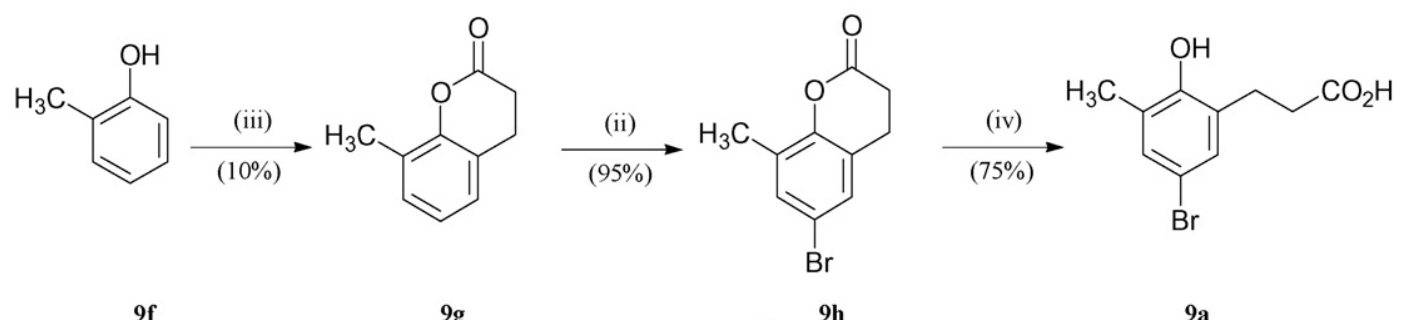<smiles>Cc1ccccc1O</smiles>

$9 \mathrm{~g}$<smiles>Cc1cc(Br)ccc1O</smiles>

(iii) $(10 \%)$ 9i

Reagents: (i) $\mathrm{H}_{2}, \mathrm{Pd} / \mathrm{C}$; (ii) $\mathrm{Br}_{2}$, glacial acetic acid (or $\mathrm{Cl}_{2} \mathrm{CH}_{2} \mathrm{CH}_{2} \mathrm{Cl}_{2}$ or $\mathrm{CH}_{2} \mathrm{C}_{12}$ ); (iii) $\mathrm{CH}_{2}=\mathrm{CH}-\mathrm{CN}, \mathrm{AlCl}_{3}$ (or $\mathrm{AlBr}_{3}$ ), $\mathrm{HCl}$ (gas), $\mathrm{C}_{6} \mathrm{H}_{4} \mathrm{Cl}_{2}$; (iv) $\mathrm{NaOH}\left(2.5 \mathrm{M}\right.$ ), $\mathrm{MeOH}: \mathrm{H}_{2} \mathrm{O}$ (1:1), $10 \% \mathrm{HCl}$;

Scheme 3. Syntheses of substituted 3-(2-hydroxyphenyl)propanoic acids.

\subsubsection{3-(2-Hydroxyphenyl)propanoic acid (6a)}

2-Hydroxycinnamic acid $\mathbf{6 f}(1.002 \mathrm{~g}, 6.10 \mathrm{mmol})$ was dissolved in absolute ethanol $(20 \mathrm{~mL})$. After addition of the catalyst, $\mathrm{Pd} / \mathrm{C}$ ( $10 \%, 50 \mathrm{mg}$ ), hydrogenation was conducted for $2 \mathrm{~h}$ under atmospheric pressure of hydrogen, after which time TLC analysis (ethyl acetate/hexane, 20:80) showed the reaction to be complete. The catalyst was removed by filtration and the ethanol was evaporated under vacuum, affording $6 \mathbf{a}$ (1.001 g, 99\%), $\mathrm{mp} \mathrm{89-90}{ }^{\circ} \mathrm{C}$, (lit. [5] mp 83-85 ${ }^{\circ} \mathrm{C}$ ). IR $v_{\max }(\mathrm{KBr}) 3400,3350,1700 ;{ }^{1} \mathrm{H}$ NMR $\left(\mathrm{DMSO}-\mathrm{d}_{6}\right) \delta$ $10.00(\mathrm{~b}, 1 \mathrm{H})$, ca. $7.0(\mathrm{~m}, 4 \mathrm{H}), 2.78(\mathrm{t}, 7 \mathrm{~Hz}, 2 \mathrm{H}), 2.50(\mathrm{t}, 7 \mathrm{~Hz}, 2 \mathrm{H}) ;{ }^{13} \mathrm{C}$ NMR $\left(\right.$ DMSO-d $\left._{6}\right) \delta 174.3,155.2,129.7,127.1,126.9,118.9,114.9,33.7$, 25.6; EI-MS $m / z 166$ [ $\left.\mathrm{M}^{+}, 18 \%\right], 148,\left[\left(\mathrm{M}-\mathrm{H}_{2} \mathrm{O}\right)^{+}, 82\right.$ ], 120 [100], 107 [25], 91 [70].

\subsubsection{Benzyl 3-(2-hydroxyphenyl)propanoate (6b)}

A solution of anhydrous benzyl alcohol $(6.20 \mathrm{~mL}, 6.514 \mathrm{~g}$, $60.2 \mathrm{mmol}), \mathbf{6 a}(1.011 \mathrm{~g}, 6.01 \mathrm{mmol})$ and trifluoroacetic anhydride $(4.80 \mathrm{~mL}, 7.085 \mathrm{~g}, 33.7 \mathrm{mmol})$ was heated at $60^{\circ} \mathrm{C}$ for $3 \mathrm{~h}$, then cooled and poured into $\mathrm{NaHCO}_{3}(50 \mathrm{~mL}$ of $8 \%$ aq.). The mixture was extracted with chloroform $(50 \mathrm{~mL} \times 3)$ and the solvent was evaporated from the combined extracts to leave a syrup ( $1.801 \mathrm{~g}$ ). This residue was subjected to chromatography using a chromatatron [ethyl acetate:hexane $(1: 8 ; 1: 4 ; 1: 2$ sequentially)]. After evaporation of the solvent from the appropriate combined fractions, $\mathbf{6 b}$ was obtained as a pure colourless oil (1.401 g, 90\%). IR $v_{\max }$ (film) 3400 , $1740 ;{ }^{1} \mathrm{H} \mathrm{NMR}\left(\mathrm{CDCl}_{3}\right) \delta 6.79-7.37(\mathrm{~m}, 9 \mathrm{H}), 5.08(\mathrm{~s}, 2 \mathrm{H}), 2.92(\mathrm{t}, 7 \mathrm{~Hz}$,
$2 \mathrm{H}), 2.74(\mathrm{t}, 7 \mathrm{~Hz}, 2 \mathrm{H}) ;{ }^{13} \mathrm{C} \mathrm{NMR}\left(\mathrm{CDCl}_{3}\right) \delta 175.1,154.2,135.4,130.5$, $128.5,128.3,128.0,127.1,120.7,116.9,116.7,66.9,34.9,25.0$; EI-MS $\mathrm{m} / z 256\left[\mathrm{M}^{+}, 100 \%\right], 239\left[(\mathrm{M}-\mathrm{OH})^{+}, 40\right], 179\left[\left(\mathrm{M}-\mathrm{C}_{6} \mathrm{H}_{5}\right)^{+}, 41\right], 148$ [40], 120 [32], 91 [99]; Anal. Calcd. for $\mathrm{C}_{16} \mathrm{H}_{16} \mathrm{O}_{3}$ : C, 74.98\%; H. 6.29\%; Found: C. $74.88 \%$ : H. $6.43 \%$.

\subsubsection{2-(2-Benzyloxycarbonylethyl)phenyl} acridine-9-carboxylate $(\mathbf{6 c})$

A mixture of $\mathbf{6 b} \quad(594 \mathrm{mg}, \quad 2.319 \mathrm{mmol})$ and 4dimethylaminopyridine ( $35 \mathrm{mg}, 0.29 \mathrm{mmol}$ ) in anhydrous pyridine ( $10 \mathrm{~mL}$, previously stored over $3 \mathrm{~A}$ molecular sieves) was heated at $90^{\circ} \mathrm{C}$ for $30 \mathrm{~min}$. The solution was cooled and pipetted into a flask containing dried acridine-9-carbonyl chloride [1] $(560 \mathrm{mg}$, $2.319 \mathrm{mmol}$ ). The reaction mixture was stirred at $100^{\circ} \mathrm{C}$ for $24 \mathrm{~h}$, then at $20^{\circ} \mathrm{C}$ for $24 \mathrm{~h}$. After removal of solvent, the crude product was dissolved in $\mathrm{CHCl}_{3}(150 \mathrm{~mL})$. The solution was washed with water $(70 \mathrm{~mL} \times 3)$, dried with anhydrous $\mathrm{Na}_{2} \mathrm{SO}_{4}$ and evaporated. The product was recrystallized from $\mathrm{CHCl}_{3} /$ hexane, and $\mathbf{6 c}$ was obtained as yellow-white crystals (909 mg, 85\%), mp $135-136^{\circ} \mathrm{C}$. (Note: without the catalyst, 4-dimethylaminopyridine, the yield was only 15\%). IR $v_{\max }(\mathrm{KBr}) 1730,1700 ;{ }^{1} \mathrm{H} \mathrm{NMR}\left(\mathrm{CDCl}_{3}\right) \delta$ $8.31(\mathrm{~d}, 9 \mathrm{~Hz}, 2 \mathrm{H}), 8.25(\mathrm{~d}, 8 \mathrm{~Hz}, 2 \mathrm{H}), 7.83(\mathrm{~m}, 2 \mathrm{H}), 7.58(\mathrm{~m}, 2 \mathrm{H})$, 7.19-7.56 (m, 9H), $5.03(\mathrm{~s}, 2 \mathrm{H}), 3.10(\mathrm{t}, 7 \mathrm{~Hz}, 2 \mathrm{H}), 2.70(\mathrm{t}, 7 \mathrm{~Hz}, 2 \mathrm{H})$; ${ }^{13} \mathrm{C}$ NMR $\left(\mathrm{CDCl}_{3}\right) 172.1,166.1,148.8,148.6,135.7,132.3,130.6$, 130.4, 130.3, 130.1, 128.5, 128.2, 128.1, 127.9, 127.6, 127.0, 124.7, $122.4,122.2,66.3,34.3,25.9$; FAB-MS $m / z 462\left[(\mathrm{M}+1)^{+}, 94 \%\right], 371$ 
[(M- $\left.\left.\mathrm{CH}_{2} \mathrm{C}_{6} \mathrm{H}_{5}\right)^{+}, 6\right], 224$ [14], 206 [23], 179 [100]; Anal. Calcd. for $\mathrm{C}_{30} \mathrm{H}_{23} \mathrm{NO}_{4}$ : C, 78.08\%; H, 5.02\%; N, 3.04\%; Found: C, 78.42\%; H, $5.18 \% ; \mathrm{N}, 3.10 \%$

\subsubsection{2-(2-Carboxyethyl)phenyl acridine-9-carboxylate (6d)}

A mixture of $\mathbf{6 c}(1.012 \mathrm{~g}, 2.17 \mathrm{mmol})$, glacial acetic acid $(16 \mathrm{~mL})$ and $48 \%$ hydrobromic acid $(4 \mathrm{~mL})$ was heated at $100^{\circ} \mathrm{C}$ for $3 \mathrm{~h}$ and then cooled. The reaction mixture was added to water $(300 \mathrm{~mL})$ and extracted with $20 \% \mathrm{MeOH} / \mathrm{CHCl}_{3}(100 \mathrm{~mL} \times 3)$. The organic extracts were combined and evaporated. The residue was suspended in chloroform and neutralized with a slight excess of triethylamine. The mixture was washed with water $(70 \mathrm{~mL} \times 3)$, and the organic layer dried $\left(\mathrm{Na}_{2} \mathrm{SO}_{4}\right)$ and evaporated. The residue was purified by recrystallization from $\mathrm{CHCl}_{3}$ to give $\mathbf{6 d}(725 \mathrm{mg}, 90 \%), \mathrm{mp} 231-232{ }^{\circ} \mathrm{C}$. IR $v_{\max }(\mathrm{KBr}) 3450,1750,1720 ;{ }^{1} \mathrm{H}$ NMR $\left(\mathrm{DMSO}^{\left.-\mathrm{d}_{6}\right)} \delta 12.41\right.$ (b, 1H), $8.37(\mathrm{~d}, 9 \mathrm{~Hz}, 2 \mathrm{H}), 8.35(\mathrm{~d}, 8 \mathrm{~Hz}, 2 \mathrm{H}), 8.03(\mathrm{~m}, 2 \mathrm{H}), 7.85(\mathrm{~m}, 2 \mathrm{H})$, $7.76(\mathrm{~d}, 8 \mathrm{~Hz}, 1 \mathrm{H}), \mathrm{ca} .7 .5(\mathrm{~m}, 3 \mathrm{H}), 3.08(\mathrm{t}, 7 \mathrm{~Hz}, 2 \mathrm{H}), 2.73(\mathrm{t}, 7 \mathrm{~Hz}$, $2 \mathrm{H}$ ); ${ }^{13} \mathrm{C}$ NMR (DMSO-d 6 ) $\delta 173.4,165.6,148.3,148.0,135.1,132.6$, 130.8, 130.3, 129.7, 128.1, 127.8, 127.0, 124.5, 122.47, 121.44, 33.8, 24.9; FAB-MS $m / z 372$ [(M+H $)^{+}, 100 \%$ ], 224 [4], 206 [40], 179 [22]; Anal. Calcd. for $\mathrm{C}_{23} \mathrm{H}_{17} \mathrm{NO}_{4}$ : C, 74.38\%; $\mathrm{H}, 4.61 \%$; N, 3.77\%; Found: C, 74.41\%; H, 4.44\%; N, 3.51\%.

\subsubsection{2-(2-Succinimidyloxycarbonylethyl)phenyl \\ acridine-9-carboxylate $(\mathbf{6 e})$}

A solution of $\mathbf{6 d}(1.001 \mathrm{~g}, 2.70 \mathrm{mmol})$ in $\mathrm{DMF}(50 \mathrm{~mL})$ was cooled in ice for $10 \mathrm{~min}$. A solution of $N, N^{\prime}$-dicyclohexylcarbodiimide $(613 \mathrm{mg}, 2.97 \mathrm{mmol})$ in DMF $(10 \mathrm{~mL})$ was added and the mixture was stirred in an ice bath for $30 \mathrm{~min}$. A solution of $\mathrm{N}$-hydroxysuccininimide (342 $\mathrm{mg}, 2.97 \mathrm{mmol}$ ) in DMF (10 mL) was added. The solution was stirred at $20^{\circ} \mathrm{C}$ overnight then evaporated to dryness. The residue was extracted with dichloromethane (DCM, $50 \mathrm{~mL}$ ) and filtered. The filtrate was evaporated to give a crude product $(1.502 \mathrm{~g})$ that was recrystallized from $\mathrm{CHCl}_{3}$ to yield pure $\mathbf{6 e}$ (1.011 g, 80\%), mp $180^{\circ} \mathrm{C}$. IR $v_{\max }(\mathrm{KBr}) 3000,1780,1720,1650$; ${ }^{1} \mathrm{H} \mathrm{NMR}\left(\mathrm{CDCl}_{3}\right) \delta 8.30(\mathrm{~d}, 9 \mathrm{~Hz}, 2 \mathrm{H}), 8.22(\mathrm{~d}, 8 \mathrm{~Hz}, 2 \mathrm{H}), 7.85(\mathrm{~m}$, $2 \mathrm{H}), 7.68(\mathrm{~m}, 2 \mathrm{H}), 7.53(\mathrm{~d}, 8 \mathrm{~Hz}, 1 \mathrm{H}), c a .7 .4(\mathrm{~m}, 2 \mathrm{H}), 7.33(\mathrm{~d}, 8 \mathrm{~Hz}$, $1 \mathrm{H}), 3.18(\mathrm{t}, 7 \mathrm{~Hz}, 2 \mathrm{H}), 2.93(\mathrm{t}, 7 \mathrm{~Hz}, 2 \mathrm{H}), 2.74(\mathrm{~s}, 4 \mathrm{H}) ;{ }^{13} \mathrm{C}$ NMR $\left(\mathrm{CDCl}_{3}\right) \delta 168.9,167.6,166.0,148.8,148.6,135.4,131.0,130.5,130.48$, 130.1, 128.4, 127.8, 127.2, 124.5, 122.4, 31.1, 27.8, 25.5; FAB-MS m/z $469\left[(\mathrm{M}+1)^{+}, 100 \%\right], 225$ [10], 206 [21], 179 [10]; Anal. Calcd. For $\mathrm{C}_{27} \mathrm{H}_{20} \mathrm{~N}_{2} \mathrm{O}_{6}$ : C, 69.28\%; H, 4.31\%; N, 5.98\%; Found: C, 69.30\%; H, $4.09 \%$; N, 5.99\%.

\subsubsection{2-(2-Succinimidyloxycarbonylethyl)phenyl \\ 10-methylacridinium-9-carboxylate trifluoromethanesulfonate \\ (6)}

To a solution of $6 \mathbf{e}(400 \mathrm{mg}, 85.45 \mathrm{mmol})$ in DCM $(10 \mathrm{~mL})$ was added $\mathrm{CF}_{3} \mathrm{SO}_{3} \mathrm{Me}(0.680 \mathrm{~mL}, 0.995 \mathrm{~g}, 41.86 \mathrm{mmol})$ under argon. After $1 \mathrm{~h}$ stirring, the product precipitated as a bright yellow solid. The mixture was stirred for $24 \mathrm{~h}$. The precipitate was filtered, washed with benzene and $\mathrm{CHCl}_{3}$ and dried to give $\mathbf{6}(500 \mathrm{mg}, 92 \%)$ as a yellow solid, $\mathrm{mp} 200-201{ }^{\circ} \mathrm{C}$. IR $v_{\max }(\mathrm{KBr}) 1840,1800,1760$; ${ }^{1} \mathrm{H}$ NMR (DMSO- $\left.\mathrm{d}_{6}\right) \delta 8.98(\mathrm{~d}, 9 \mathrm{~Hz}, 2 \mathrm{H}), \mathrm{ca} .8 .6(\mathrm{~m}, 4 \mathrm{H}), 8.18(\mathrm{~m}$, $2 \mathrm{H}), 7.94(\mathrm{~d}, 8 \mathrm{~Hz}, 1 \mathrm{H}), 7.63(\mathrm{~d}, 8 \mathrm{~Hz}, 1 \mathrm{H}), 7.54(\mathrm{~m}, 1 \mathrm{H}), 7.48(\mathrm{~m}, 1 \mathrm{H})$, $4.99(\mathrm{~s}, 3 \mathrm{H}), 3.09$ (apparent s, 4H), 2.81 (s, 4H); ${ }^{13} \mathrm{C} \mathrm{NMR}\left(\right.$ DMSO-d $\left._{6}\right)$ $\delta 170.1,168.2,166.2,147.8,146.6,141.9,139.3,131.0,130.5,129.9$, $128.3,127.6,127.1,122.5,122.4,120.0,39.8,29.9,25.4,23.9\left(\mathrm{CF}_{3}\right.$ signal not observed); FAB-MS $m / z 483$ [( $\left(\mathrm{M}-\mathrm{SO}_{3} \mathrm{CF}_{3}\right)^{+}, 100 \%$ ], 221 [16], 193 [37]; Anal. Calcd. for $\mathrm{C}_{29} \mathrm{H}_{23} \mathrm{~N}_{2} \mathrm{~F}_{3} \mathrm{O}_{9} \mathrm{~S}$ : C, 55.06\%; H, 3.67\%; N, 4.43\%; Found: C, 54.98\%; H, 3.86\%; N, 4.23\%.

\subsubsection{3-(3,5-Dimethyl-2-hydroxyphenyl) propanoic acid lactone (7g)}

2,4-Dimethylphenol (7f, $2.441 \mathrm{~g}, 0.0200 \mathrm{~mol}$ ) was dissolved in acrylonitrile $(1.30 \mathrm{~mL}, 1.048 \mathrm{~g}, 0.0200 \mathrm{~mol})$. Anhydrous $\mathrm{AlCl}_{3}$
(5.341 $\mathrm{g}, 0.0400 \mathrm{~mol})$ and 1,2-dichlorobenzene $(10 \mathrm{~mL})$ were added and dry $\mathrm{HCl}$ gas was bubbled through at $110^{\circ} \mathrm{C}$ for $1 \mathrm{~h}$. The mixture was added to water $(100 \mathrm{~mL})$ and the whole was extracted with $\mathrm{CHCl}_{3}(70 \mathrm{~mL} \times 3)$. The combined extract was washed with sat. $\mathrm{NaCl}$ aq., dried over $\mathrm{Na}_{2} \mathrm{SO}_{4}$ and evaporated to give a yellow oil $(4.002 \mathrm{~g})$, which was subjected to chromatography by chromatatron (EtOAc:hexane $=1: 8 ; 1: 4 ; 1: 2$ sequentially) to give $\mathbf{7 g}(3.061 \mathrm{~g}$, $88 \%)$ as a yellow gum. ${ }^{1} \mathrm{H} \mathrm{NMR}\left(\mathrm{CDCl}_{3}\right) \delta 6.89(\mathrm{~s}, 1 \mathrm{H}), 6.88(\mathrm{~s}, 1 \mathrm{H})$, $2.90(\mathrm{t}, 8 \mathrm{~Hz}, 2 \mathrm{H}), 2.72(\mathrm{t}, 8 \mathrm{~Hz}, 2 \mathrm{H}), 2.26(\mathrm{~s}, 3 \mathrm{H}), 2.25(\mathrm{~s}, 3 \mathrm{H}) ;{ }^{13} \mathrm{C}$ $\operatorname{NMR}\left(\mathrm{CDCl}_{3}\right) \delta 169.1,148.2,133.4,131.5,130.3,127.2,125.9,29.3$, 23.9, 20.6, 15.5; EI-MS $m / z 176$ [ $\left.\mathrm{M}^{+}, 100 \%\right], 148$ [(M-CO $\left.)^{+}, 90\right], 134$ [70], 106 [50], 91 [90], 77 [40]; Anal. Calcd. for $\mathrm{C}_{11} \mathrm{H}_{12} \mathrm{O}_{2}$ : C, 74.98\%; H, 6.86\%; Found: C, 74.74\%; H, 6.99\%.

\subsubsection{3-(3,5-Dimethyl-2-hydroxyphenyl)propanoic acid (7a)}

To $7 \mathbf{g}(1.001 \mathrm{~g}, 5.68 \mathrm{mmol})$ in $\mathrm{MeOH}: \mathrm{H}_{2} \mathrm{O}(1: 1,25 \mathrm{~mL})$ was added dropwise $\mathrm{NaOH}(227 \mathrm{mg}, 5.68 \mathrm{mmol})$ in $\mathrm{H}_{2} \mathrm{O}(2.5 \mathrm{~mL})$. The mixture was stirred at $50^{\circ} \mathrm{C}$ for $10 \mathrm{~h}$ and $20^{\circ} \mathrm{C}$ overnight, then evaporated. The residue was washed with acetone and air dried to give a white salt $(1.301 \mathrm{~g})$, which was dissolved in water and acidified with $10 \%$ $\mathrm{HCl}$ aq. to $\mathrm{pH}$ 6. The solution was extracted with $\mathrm{CHCl}_{3}(70 \mathrm{~mL} \times 3)$. The combined extracts were washed with sat. $\mathrm{NaCl}$ aq., dried over $\mathrm{Na}_{2} \mathrm{SO}_{4}$ and evaporated to give a yellow oil ( $1.401 \mathrm{~g}$ ), which crystallized from $\mathrm{CHCl}_{3} /$ hexane to give a white solid (7a, $880 \mathrm{mg}, 80 \%$ ), $\mathrm{mp}$ 92-93 ${ }^{\circ} \mathrm{C}$. IR $v_{\max }(\mathrm{KBr}) 3543,3450,1706 ;{ }^{1} \mathrm{H} \mathrm{NMR}\left(\mathrm{CDCl}_{3}\right) \delta 6.80$ $(\mathrm{s}, 1 \mathrm{H}), 6.74(\mathrm{~s}, 1 \mathrm{H}), 2.85(\mathrm{t}, 8 \mathrm{~Hz}, 2 \mathrm{H}), 2.72(\mathrm{t}, 8 \mathrm{~Hz}, 2 \mathrm{H}), 2.22(\mathrm{~s}, 3 \mathrm{H})$, $2.19(\mathrm{~s}, 3 \mathrm{H})$, (exchangeable proton signals too broad to identify); ${ }^{13} \mathrm{C}$ $\operatorname{NMR}\left(\mathrm{CDCl}_{3}\right) \delta 180.6,149.7,129.9,129.7,128.4,126.2,124.8,34.8$, 24.6, 20.4, 16.1; EI-MS $m / z 194\left[\mathrm{M}^{+}, 100 \%\right], 177$ [(M- $\left.\left.\mathrm{H}_{2} \mathrm{O}+1\right)^{+}, 45\right]$, 148 [12], 57 [30]; Anal. Calcd. for $\mathrm{C}_{11} \mathrm{H}_{14} \mathrm{O}_{3} ; \mathrm{C}, 68.02 \%$; $\mathrm{H}, 7.27 \%$; Found: C, $68.12 \%$; H. 7.13\%.

\subsubsection{Benzyl 3-(3,5-dimethyl-2-hydroxyphenyl)propanoate (7b)}

The procedure was analogous to that used to prepare $\mathbf{6 b}$ except for the different starting material. Pure $\mathbf{7 b}$ was obtained as a colourless oil in $81 \%$ yield. ${ }^{1} \mathrm{H}$ NMR $\left(\mathrm{CDCl}_{3}\right) \delta \mathrm{ca} .7 .3(\mathrm{~m}, 5 \mathrm{H}), 6.81(\mathrm{~s}, 1 \mathrm{H})$, $6.73(\mathrm{~s}, 1 \mathrm{H}), 6.72(\mathrm{~s}, 1 \mathrm{H}), 5.09(\mathrm{~s}, 2 \mathrm{H}), 2.80(\mathrm{~m}, 2 \mathrm{H}), 2.70(\mathrm{~m}, 2 \mathrm{H})$, $2.22(\mathrm{~s}, 3 \mathrm{H}), 2.20(\mathrm{~s}, 3 \mathrm{H}) ;{ }^{13} \mathrm{C}$ NMR $\left(\mathrm{CDCl}_{3}\right) \delta 175.5,150.2,135.4$, 130.3, 129.4, 128.6, 128.5, 128.4, 128.3, 126.4, 125.6, 67.6, 35.4, 24.6, 20.4, 16.6; EI-MS m/z 284 [ $\mathrm{M}^{+}, 80 \%$ ], 267 [40], 194 [60], 176 [30], 148 [50], 91 [100]; Anal. Calcd. for $\mathrm{C}_{18} \mathrm{H}_{20} \mathrm{O}_{3}+1 / 4 \mathrm{H}_{2} \mathrm{O}: \mathrm{C}, 74.85 \% ; \mathrm{H}$, 7.15\%; Found: C, 74.96\%; H, 7.27\%.

\subsubsection{4,6-Dimethyl-2-(2-benzyloxycarbonylethyl)phenyl acridine-9-carboxylate (7c)}

The preparation was analogous to that used to prepare $\mathbf{6 c}$. 7c was obtained as a yellow gum in $70 \%$ yield. ${ }^{1} \mathrm{H} \mathrm{NMR}\left(\mathrm{CDCl}_{3}\right) \delta \mathrm{ca}$. $8.4(\mathrm{~m}, 4 \mathrm{H}), 7.84$ (apparent t, $8 \mathrm{~Hz}, 2 \mathrm{H}), 7.60(\mathrm{~d}, 8 \mathrm{~Hz}, 2 \mathrm{H}), c a .7 .3$ (m, 5H), $7.04(\mathrm{~s}, 1 \mathrm{H}), 7.02(\mathrm{~s}, 1 \mathrm{H}), 5.05(\mathrm{~s}, 2 \mathrm{H}), 3.10(\mathrm{t}, 7.5 \mathrm{~Hz}, 2 \mathrm{H})$, $2.72(\mathrm{t}, 7.5 \mathrm{~Hz}, 2 \mathrm{H}), 2.39(\mathrm{~s}, 3 \mathrm{H}), 2.33(\mathrm{~s}, 3 \mathrm{H}) ;{ }^{13} \mathrm{C} \mathrm{NMR}\left(\mathrm{CDCl}_{3}\right) \delta$ $172.3,165.6,148.3,145.7,136.6,135.7,132.0,130.7,130.6,130.1$, 129.6, 129.1, 128.6, 128.5, 128.2, 128.1, 127.7, 127.2, 125.1, 66.4, 34.5, 26.3, 20.8, 18.0; FAB-MS $m / z 490$ [(M+1 $)^{+}, 100 \%$ ], 461 [7], 400 [20], 224 [8]; Anal Calcd. for $\mathrm{C}_{32} \mathrm{H}_{27} \mathrm{NO}_{4}+1 / 2 \mathrm{H}_{2} \mathrm{O}$ : C, $77.09 \%$; $\mathrm{H}, 5.66 \%$; N, 2.81\%; Found: C, 77.15\%; H, 5.66\%; N, 2.80\%.

\subsubsection{4,6-Dimethyl-2-(2-carboxyethyl)phenyl} acridine-9-carboxylate (7d)

The procedure was similar to that used to prepare $6 \mathbf{d}$. 7d was obtained in $80 \%$ yield, $\mathrm{mp} 189-190^{\circ} \mathrm{C}$. IR $v_{\max }(\mathrm{KBr}) 3450,1750$, 1713; ${ }^{1} \mathrm{H}$ NMR (DMSO-d 6 ) (acidic proton not observed) $\delta \mathrm{ca} .8 .3(\mathrm{~m}$, $4 \mathrm{H}), 8.00(\mathrm{~m}, 2 \mathrm{H}), 7.80(\mathrm{~m}, 2 \mathrm{H}), 7.02(\mathrm{~s}, 1 \mathrm{H}), 7.00(\mathrm{~s}, 1 \mathrm{H}), 2.90(\mathrm{t}$, $7.5 \mathrm{~Hz}, 2 \mathrm{H}), 2.50(\mathrm{t}, 7.5 \mathrm{~Hz}, 2 \mathrm{H}), 2.54(\mathrm{~s}, 3 \mathrm{H}), 2.38(\mathrm{~s}, 3 \mathrm{H}),{ }^{3} \mathrm{C} \mathrm{NMR}$ $\left(\right.$ DMSO $\left.^{-} \mathrm{d}_{6}\right) \delta 173.3,165.0,148.0,145.2,135.9,134.8,132.3,130.7$, 130.0, 129.8, 129.4, 128.2, 128.0, 124.6, 121.7, 33.8, 25.6, 20.3, 17.2; FAB-MS $m / z 400\left[(\mathrm{M}+1)^{+}, 100 \%\right], 356\left[\left(\mathrm{M}+1-\mathrm{CO}_{2}\right)^{+}, 60\right], 224$ [29]; 
Anal. Calcd. for $\mathrm{C}_{25} \mathrm{H}_{21} \mathrm{NO}_{4}$ : C, 75.17\%; H, 5.23\%; N, 3.51\%; Found: C, 75.20\%; H, 5.25\%; N, 3.74\%.

\subsubsection{4,6-Dimethyl-2-(2-}

succinimidyloxycarbonylethyl)phenylacridine-9-carboxylate (7e)

The procedure was similar to that used for $\mathbf{6 e}$ and gave $7 \mathbf{e}$ in $90 \%$ yield, mp $149-150{ }^{\circ} \mathrm{C}$. IR $v_{\max }(\mathrm{KBr}) 1785,1737 ;{ }^{1} \mathrm{H} \mathrm{NMR}\left(\mathrm{CDCl}_{3}\right) \delta$ $8.39(\mathrm{~d}, 9 \mathrm{~Hz}, 2 \mathrm{H}), 8.33(\mathrm{~d}, 9 \mathrm{~Hz}, 2 \mathrm{H}), 7.86(\mathrm{~m}, 2 \mathrm{H}), 7.68(\mathrm{~m}, 2 \mathrm{H})$, 7.08 (apparent s, 2H), $3.18(\mathrm{t}, 7.5 \mathrm{~Hz}, 2 \mathrm{H}), 2.96(\mathrm{t}, 7.5 \mathrm{~Hz}, 2 \mathrm{H}), 2.80$ (s, 4H), $2.39(\mathrm{~s}, 3 \mathrm{H}), 2.38(\mathrm{~s}, 3 \mathrm{H}) ;{ }^{13} \mathrm{C}$ NMR $\delta$ 168.7, 167.6, 165.7, 148.7, 145.7, 136.8, 135.2, 131.0, 130.9, 130.3, 130.2, 130.1, 128.4, 127.7, 124.9, 127.8, 31.4, 26.9, 25.6, 20.8, 17.8; FAB-MS $m / z 497$ $\left[(\mathrm{M}+1)^{+}, 100 \%\right], 400$ [7], 225 [40], 206 [75], 179 [40]; Anal. Calcd. for $\mathrm{C}_{29} \mathrm{H}_{24} \mathrm{~N}_{2} \mathrm{O}_{6}$ : C, 70.15\%; H, 4.87\%; N, 5.64\%; Found: C, 69.89\%; H, $4.96 \%$; N, 5.53\%.

\subsubsection{4,6-Dimethyl-2-(2-succinimidyloxycarbonylethyl)phenyl} 10-methylacridinium-9-carboxylate trifluoromethanesulfonate (7)

The preparation was similar to that used for $\mathbf{6}$ and gave $\mathbf{7}$ as a yellow solid in $90 \%$ yield, $\mathrm{mp} 179-180^{\circ} \mathrm{C}$. IR $\nu_{\max }(\mathrm{KBr}) 1820,1800$, 1750; ${ }^{1} \mathrm{H}$ NMR (DMSO-d 6 ) $\delta 8.97(\mathrm{~d}, 9.0 \mathrm{~Hz}, 2 \mathrm{H}), 8.52(\mathrm{~m}, 4 \mathrm{H}), 8.17$ (m, 2H), $7.23(\mathrm{~s}, 1 \mathrm{H}), 7.16(\mathrm{~s}, 1 \mathrm{H}), 4.99(\mathrm{~s}, 3 \mathrm{H}), 3.08$ (app s, 4H), 2.80 $(\mathrm{s}, 4 \mathrm{H}), 2.46(\mathrm{~s}, 3 \mathrm{H}), 2.39(\mathrm{~s}, 3 \mathrm{H}) ;{ }^{13} \mathrm{C}$ NMR $\left(\mathrm{DMSO}^{-\mathrm{d}_{6}}\right) \delta 169.9,168.1$, 162.7, 146.6, 144.7, 141.8, 139.1, 136.8, 130.8, 130.7, 129.7, 129.6, $128.2,127.0,126.9,122.5,120.0,39.9,29.9,25.3,24.7,20.3,17.4$; FABMS $m / z 511$ [(M- $\left.\mathrm{SO}_{3} \mathrm{CF}_{3}\right)^{+}, 100 \%$ ], 428 [20], 414 [5], 221 [58], 207 [7], 193 [61]; Anal. Calcd. for $\mathrm{C}_{31} \mathrm{H}_{27} \mathrm{~N}_{2} \mathrm{~F}_{3} \mathrm{O}_{9} \mathrm{~S}$ : C, 56.36\%; $\mathrm{H}, 4.12 \%$; N, 4.24\%; Found: C, 56.37\%; H, 4.38\%; N, 4.04\%.

\subsubsection{3-(3,5-Dibromo-2-hydroxyphenyl)propanoic acid (8a)}

To 3-(2-hydroxyphenyl)propanoic acid ( $882 \mathrm{mg}, 5.31 \mathrm{mmol}$ ) in glacial $\mathrm{AcOH}(50 \mathrm{~mL})$ was added $\mathrm{Br}_{2}(1.701 \mathrm{~g}, 0.54 \mathrm{~mL}, 10.62 \mathrm{mmol})$ and the mixture stirred in the dark for $72 \mathrm{~h}$. Evaporation in vacuo left a residue $(2.011 \mathrm{~g})$. This was dissolved in $\mathrm{CHCl}_{3}(50 \mathrm{~mL})$, washed with sat. $\mathrm{NaCl}(40 \mathrm{~mL} \times 3)$, dried $\left(\mathrm{Na}_{2} \mathrm{SO}_{4}\right)$ and evaporated to a white powder $(1.901 \mathrm{~g})$. Recrystallization from $\mathrm{CHCl}_{3}$ gave white needles of $8 \mathbf{a}(1.551 \mathrm{~g}, 90 \%), \mathrm{mp} 105^{\circ} \mathrm{C}$. IR $v_{\max }(\mathrm{KBr}) 3500,3000,1740$; ${ }^{1} \mathrm{H}$ NMR (DMSO-d 6 ) $\delta$ 12.0-12.4 (b, 1H), 9.38 (s, 1H), 7.53 (d, $2 \mathrm{~Hz}$, 1H), $7.29(\mathrm{~d}, 2 \mathrm{~Hz}, 1 \mathrm{H}), 2.85(\mathrm{t}, 7.5 \mathrm{~Hz}, 2 \mathrm{H}), 2.52(\mathrm{t}, 7.5 \mathrm{~Hz}, 2 \mathrm{H}) ;{ }^{13} \mathrm{C}$ NMR (DMSO-d $\left.\mathrm{d}_{6}\right) \delta 173.8,151.2,132.4,132.2,131.7,112.2,111.0,32.2$, 25.8; EI-MS $m / z 326\left[\mathrm{M}^{+}\left({ }^{81} \mathrm{Br}_{2}\right), 35 \%\right] 324$ [ $\left.\mathrm{M}^{+}\left({ }^{81} \mathrm{Br}^{79} \mathrm{Br}\right), 70\right], 322$ $\left[\mathrm{M}^{+}\left({ }^{79} \mathrm{Br}_{2}\right), 35\right], 306$ [80], 278 [100]; Anal. Calcd. for $\mathrm{C}_{9} \mathrm{H}_{8} \mathrm{Br}_{2} \mathrm{O}_{3}: \mathrm{C}$, 33.37\%; H, 2.49\%; Found: C, 33.19\%; H, 2.59\%.

\subsubsection{Benzyl 3-(3,5-dibromo-2-hydroxyphenyl)propanoate (8b)}

The preparation was similar to that used to prepare $\mathbf{6 b}$. $\mathbf{8 b}$ was obtained as a colourless oil in a yield of $88 \%$. IR $v_{\max }(\mathrm{KBr}) 3500$, $1750 ;{ }^{1} \mathrm{H} \mathrm{NMR}\left(\mathrm{CDCl}_{3}\right) \delta 7.45(\mathrm{~d}, 2 \mathrm{~Hz}, 1 \mathrm{H}), 7.34(\mathrm{~m}, 5 \mathrm{H}), 7.18(\mathrm{~d}, 2 \mathrm{~Hz}$, $1 \mathrm{H}), 6.04(\mathrm{~s}, 1 \mathrm{H}), 5.11(\mathrm{~s}, 2 \mathrm{H}), 2.94(\mathrm{t}, 7.5 \mathrm{~Hz}, 2 \mathrm{H}), 2.70(\mathrm{t}, 7.5 \mathrm{~Hz}, 2 \mathrm{H})$; ${ }^{13} \mathrm{C} \mathrm{NMR}\left(\mathrm{CDCl}_{3}\right) \delta 173.4,149.9,135.5,132.5,132.4,130.0,128.6$, $128.5,128.3,112.3,111.5,66.7,35.9,25.9$; EI-MS $m / z 306$ [5\%], 278 [2], 91 [100]; CI-MS $434\left[\left(\mathrm{M}+\mathrm{NH}_{4}\right)^{+},\left({ }^{81} \mathrm{Br}_{2}\right), 20 \%\right], 432\left[\left(\mathrm{M}+\mathrm{NH}_{4}\right)^{+}\right.$, $\left.\left({ }^{81} \mathrm{Br}^{79} \mathrm{Br}\right), 40\right], 430\left[\left(\mathrm{M}+\mathrm{NH}_{4}\right)^{+},\left({ }^{79} \mathrm{Br}_{2}\right), 20\right], 415\left[(\mathrm{M}+1)^{+}, 15\right], 324$ [8], 306 [5], 278 [2], 108 [100], 91 [52]; Anal. Calcd. for $\mathrm{C}_{16} \mathrm{H}_{14} \mathrm{Br}_{2} \mathrm{O}_{3}$ : C, $46.40 \%$; H, 3.41\%: Found: C, 46.23\%; H, 3.22\%.

\subsubsection{4,6-Dibromo-2-(2-benzyloxycarbonylethyl)phenyl}

\section{acridine-9-carboxylate $(\mathbf{8 c})$}

The procedure was analogous to that used for $\mathbf{6 c}$ and gave $8 \mathbf{c}$ as a yellow gum (85\% yield). ${ }^{1} \mathrm{H} \mathrm{NMR}\left(\mathrm{CDCl}_{3}\right) \delta 8.70(\mathrm{~d} .9 \mathrm{~Hz}, 2 \mathrm{H}), 8.30(\mathrm{~d}$, $8 \mathrm{~Hz}, 2 \mathrm{H}), 7.80(\mathrm{~m}, 2 \mathrm{H}), 7.74(\mathrm{~d}, 3 \mathrm{~Hz}, 1 \mathrm{H}), 7.60(\mathrm{~m}, 2 \mathrm{H}), 7.46(\mathrm{~d}, 3 \mathrm{~Hz}$, $1 \mathrm{H}), 7.25(\mathrm{~m}, 5 \mathrm{H}), 5.04(\mathrm{~s}, 2 \mathrm{H}), 3.10(\mathrm{t}, 8 \mathrm{~Hz}, 2 \mathrm{H}), 2.70(\mathrm{t}, 8 \mathrm{~Hz}, 2 \mathrm{H}) ;{ }^{13} \mathrm{C}$ $\operatorname{NMR}\left(\mathrm{CDCl}_{3}\right) \delta 171.4,164.4,148.6,146.2,136.7,135.4,134.4,133.7$, $132.4,130.3,130.2,128.8,128.5,128.2,128.1,127.7,125.3,120.5$,
117.8, 66.6, 33.8, 26.5; FAB-MS $m / z 622\left[(\mathrm{M}+1)^{+},\left({ }^{81} \mathrm{Br}_{2}\right), 17 \%\right], 620$ $\left[(\mathrm{M}+1)^{+},\left({ }^{79} \mathrm{Br}^{81} \mathrm{Br}\right), 35\right], 618\left[(\mathrm{M}+1)+\left({ }^{79} \mathrm{Br}_{2}\right), 17\right], 206$ [55], 179 [32]; Anal. Calcd. for $\mathrm{C}_{30} \mathrm{H}_{21} \mathrm{NBr}_{2} \mathrm{O}_{4}$ : C, 58.18\%; $\mathrm{H}, 3.42 \%$; $\mathrm{N}, 2.26 \%$; Found: C, $58.05 \%$; $\mathrm{H}, 3.32 \%$; N, 2.15\%.

\subsubsection{4,6-Dibromo-2-(2-carboxyethyl)phenyl} acridine-9-carboxylate $(\mathbf{8 d})$

The preparation was similar to that used for $\mathbf{6 d}$ and gave $8 \mathbf{d}$ in $80 \%$ yield, mp $228-230^{\circ} \mathrm{C}$. IR $v_{\max }(\mathrm{KBr}) 3500,1750,1700 ;{ }^{1} \mathrm{H}$ NMR $\left(\right.$ DMSO $\left._{6}\right) \delta 8.56(\mathrm{~d}, 9 \mathrm{~Hz}, 2 \mathrm{H}), 8.34(\mathrm{~d}, 8 \mathrm{~Hz}, 2 \mathrm{H}), 8.05(\mathrm{~d}, 3 \mathrm{~Hz}, 1 \mathrm{H})$, $8.00(\mathrm{~m}, 2 \mathrm{H}), 7.86(\mathrm{~m}, 2 \mathrm{H}), 7.82(\mathrm{~d}, 3 \mathrm{~Hz}, 1 \mathrm{H}), 3.20(\mathrm{t}, 7.5 \mathrm{~Hz}, 2 \mathrm{H})$, $2.70(\mathrm{t}, 7.5 \mathrm{~Hz}, 2 \mathrm{H})$, (the exchangeable proton was not observed); ${ }^{13}$ C NMR (DMSO-d $\left.{ }_{6}\right) \delta 173.1,164.1,148.1,145.6,137.6,133.6,133.2$, $132.5,130.9,129.9,128.4,124.8,121.8,120.1,117.2,33.1,25.7$; FABMS $m / z 530\left[(\mathrm{M}+1)^{+},\left({ }^{79} \mathrm{Br}^{81} \mathrm{Br}\right), 35 \%\right], 512\left[\left(\mathrm{M}+1-\mathrm{H}_{2} \mathrm{O}\right)^{+}, 17\right], 224$ [4], 206 [8], 179 [15]; Anal. Calcd. for $\mathrm{C}_{23} \mathrm{H}_{15} \mathrm{NBr}_{2} \mathrm{O}_{4}$ : C, 52.20\%; $\mathrm{H}$, 2.86\%; N, 2.65\%; Found: C, 52.10\%; H, 2.74\%; N, 2.80\%.

\subsubsection{4,6-Dibromo-2-(2-succinimidyloxycarbonylethyl)phenyl acridine-9-carboxylate $(\mathbf{8 e})$}

The procedure was similar to that for $\mathbf{6 e}$ and gave $\mathbf{8 e}$ in $75 \%$ yield, mp $189-190{ }^{\circ} \mathrm{C} .{ }^{1} \mathrm{H}$ NMR $\left(\mathrm{CDCl}_{3}\right) \delta 8.53(\mathrm{~d}, 9 \mathrm{~Hz}, 2 \mathrm{H}), 8.35(\mathrm{~d}$, $8 \mathrm{~Hz}, 2 \mathrm{H}), 7.84(\mathrm{~m}, 2 \mathrm{H}), 7.82$ (d, $3 \mathrm{~Hz}, 1 \mathrm{H}), 7.70(\mathrm{~m}, 2 \mathrm{H}), 7.54(\mathrm{~d}, 3 \mathrm{~Hz}$, 1H), $3.20(\mathrm{t}, 7.5 \mathrm{~Hz}, 2 \mathrm{H}), 2.95(\mathrm{t}, 7.5 \mathrm{~Hz}, 2 \mathrm{H}), 2.81(\mathrm{~s}, 4 \mathrm{H}) ;{ }^{13} \mathrm{C} \mathrm{NMR}$ $\left(\mathrm{CDCl}_{3}\right) \delta 168.6,167.5,164.5,148.8,146.4,135.7,135.1,133.7132 .5$, $130.4,128.0,125.3,123.0,120.8,118.2,31.0,26.3,25.6$; FAB-MS $m / z$ $629\left[(\mathrm{M}+1)^{+},\left({ }^{81} \mathrm{Br}_{2}\right), 10 \%\right], 627\left[(\mathrm{M}+1)^{+},\left({ }^{79} \mathrm{Br}^{81} \mathrm{Br}\right), 20\right], 625\left[(\mathrm{M}+1)^{+}\right.$, $\left({ }^{79} \mathrm{Br}_{2}\right), 10$ ], 114 [100]; Anal. Calcd. for $\mathrm{C}_{27} \mathrm{H}_{18} \mathrm{~N}_{2} \mathrm{Br}_{2} \mathrm{O}_{6}$ : C, 51.78\%; $\mathrm{H}$, 2.90\%; N, 4.47\%; Found: C, 51.71\%; H, 2.97\%; N, 4.42\%.

\subsubsection{4,6-Dibromo-2-(2-succinimidyloxycarbonylethyl)phenyl} 10-methylacridinium-9-carboxylate trifluoromethanesulfonate (8)

The preparation was similar to that for $\mathbf{6}$ and gave $\mathbf{8}$ as a yellow solid in $80 \%$ yield, $\operatorname{mp} 224-225^{\circ} \mathrm{C}$. IR $v_{\max }(\mathrm{KBr}) 1800,1760,1750 ;{ }^{1} \mathrm{H}$ NMR (DMSO-d $\left.{ }_{6}\right) \delta 8.98(\mathrm{~d}, 9 \mathrm{~Hz}, 2 \mathrm{H}), 8.76(\mathrm{~d}, 8 \mathrm{~Hz}, 2 \mathrm{H}), 8.56(\mathrm{~m}, 2 \mathrm{H})$, $8.20(\mathrm{~m}, 2 \mathrm{H}), 8.09$ (d, $3 \mathrm{~Hz}, 1 \mathrm{H}), 7.93(\mathrm{~d}, 3 \mathrm{~Hz}, 1 \mathrm{H}), 5.00(\mathrm{~s}, 3 \mathrm{H}), 3.16$ (apparent s, 4H), $2.79(\mathrm{~s}, 4 \mathrm{H}) ;{ }^{13} \mathrm{C}$ NMR (DMSO-d 6 ) $\delta 169.9,167.9$, 161.7, 145.2, 144.9, 141.8, 139.1, 136.1, 134.1, 132.6, 129.8, 128.6, 127.1, 124.6, 122.7, 120.9, 117.1, 39.8, 29.3, 25.3, 24.7; FAB-MS m/z 643 $\left[\left(\mathrm{M}-\mathrm{SO}_{3} \mathrm{CF}_{5}\right)^{+},\left({ }^{81} \mathrm{Br}_{2}\right), 50 \%\right], 641\left[\left(\mathrm{M}-\mathrm{SO}_{3} \mathrm{CF}_{3}\right)^{+},\left({ }^{79} \mathrm{Br}{ }^{81} \mathrm{Br}\right), 100\right]$, 639 [(M-SO $\left.\left.\mathrm{SO}_{3}\right)^{+},\left({ }^{79} \mathrm{Br}_{2}\right), 50\right], 627$ [14], 221 [10], 193 [76], 179 [14]; Anal. Calcd. for $\mathrm{C}_{29} \mathrm{H}_{21} \mathrm{~N}_{2} \mathrm{Br}_{2} \mathrm{~F}_{3} \mathrm{O}_{9} \mathrm{~S}$ : C, 44.07\%; $\mathrm{H}, 2.68 \%$; $\mathrm{N}$, 3.54\%; Found: C, 44.19\%; H, 2.62\%; N, 3.61\%.

\subsubsection{Synthesis of 4-bromo-2-methylphenol (9i)}

To $o$-cresol $(1.302 \mathrm{~g}, 12 \mathrm{mmol})$ in 1,2-dichloroethane $(20 \mathrm{~mL})$ was added dropwise bromine $(1.611 \mathrm{~g}, 10 \mathrm{mmol})$ in DCM $(20 \mathrm{~mL})$ at $0^{\circ} \mathrm{C}$, over $30 \mathrm{~min}$. The mixture was stirred $2 \mathrm{~h}$ at $0^{\circ} \mathrm{C}$, then $20^{\circ} \mathrm{C}$ overnight. Evaporation left a residue $(2.012 \mathrm{~g})$ that was dissolved in $\mathrm{CHCl}_{3}(70 \mathrm{~mL})$, washed with saturated $\mathrm{NaCl}$ aq. $(40 \mathrm{~mL} \times 3)$, dried $\left(\mathrm{Na}_{2} \mathrm{SO}_{4}\right)$ and evaporated to give a white powder $(1.901 \mathrm{~g})$. Recrystallization from $\mathrm{PhH} /$ hexane gave $\mathbf{9 i}$ (white needles, $1.811 \mathrm{~g}, 96 \%$ ), mp $63{ }^{\circ} \mathrm{C}$, [lit. [6] mp $63^{\circ} \mathrm{C}$ ]. IR $v_{\max }(\mathrm{KBr}) 3500 ;{ }^{1} \mathrm{H}$ NMR $\left(\mathrm{CDCl}_{3}\right)$ $\delta 7.23(\mathrm{~d}, 2 \mathrm{~Hz}, 1 \mathrm{H}), 7.15(\mathrm{dd}, 8,2 \mathrm{~Hz}, 1 \mathrm{H}), 6.22(\mathrm{~d}, 8 \mathrm{~Hz}, 1 \mathrm{H}), 4.89$ $(\mathrm{s}, 1 \mathrm{H}), 2.20(\mathrm{~s}, 3 \mathrm{H}) ;{ }^{13} \mathrm{C} \mathrm{NMR}\left(\mathrm{CDCl}_{3}\right) \delta 152.8,133.5,129.8,126.2$, 116.5, 112.6, 15.6; EI-MS 188 [ $\left.\mathrm{M}^{+},\left({ }^{81} \mathrm{Br}\right), 98 \%\right], 186$ [ $\left.\mathrm{M}^{+},\left({ }^{79} \mathrm{Br}\right), 98\right]$, $107\left[(\mathrm{M}-\mathrm{Br})^{+}, 100\right], 89$ [20], 77 [70], 51 [38].

\subsubsection{Synthesis of 3-(2-hydroxy-3-methylphenyl)propanoic acid lactone $(\mathbf{9 g})$}

$o$-Cresol $(1.012 \mathrm{~g}, 9.25 \mathrm{mmol})$ and acrylonitrile $(0.61 \mathrm{~mL}$, $9.25 \mathrm{mmol}$ ) in 1,2-dichlorobenzene (DCB, $5 \mathrm{~mL}$ ) was added dropwise to a suspension of anhydrous $\mathrm{AlCl}_{3}(1.231 \mathrm{~g}, 18.5 \mathrm{mmol})$ in DCB $(20 \mathrm{~mL})$. Dry $\mathrm{HCl}$ gas was passed into the mixture (at $120^{\circ} \mathrm{C}$ ) for $1 \mathrm{~h}$. The mixture was heated for $15 \mathrm{~h}$ at $110^{\circ} \mathrm{C}$, added 
to water $(100 \mathrm{~mL})$ and the whole was extracted with $\mathrm{CHCl}_{3}$ $(70 \mathrm{~mL} \times 3)$. The combined extracts were washed with sat. $\mathrm{NaCl}$ aq. $(70 \mathrm{~mL} \times 3)$, dried $\left(\mathrm{Na}_{2} \mathrm{SO}_{4}\right)$ and evaporated to give a yellow solid $(2.012 \mathrm{~g})$. This was chromatographed using a chromatatron (hexane:EtOAc $=100: 0,4: 1,1: 1$ sequentially) to give two pure white solids. One [Rf=0.4 in EtOAc:hexane (1:4)] was recrystallized with $\mathrm{CHCl}_{3} /$ hexane and gave white crystals of $\mathbf{9 g}(150 \mathrm{mg}$, $10 \%), \mathrm{mp} 73^{\circ} \mathrm{C}$. IR $v_{\max }(\mathrm{KBr}) 2959,1763 ;{ }^{1} \mathrm{H} \mathrm{NMR}\left(\mathrm{CDCl}_{3}\right) \delta 7.11$ (dd, 1, 9 Hz, 1H), $7.02(\mathrm{~m}, 2 \mathrm{H}), 2.97(\mathrm{t}, 7 \mathrm{~Hz}, 2 \mathrm{H}), 2.76(\mathrm{t}, 7 \mathrm{~Hz}, 2 \mathrm{H})$, $2.30(\mathrm{~s}, 3 \mathrm{H}) ;{ }^{13} \mathrm{C}$ NMR $\left(\mathrm{CDCl}_{3}\right) \delta 168.8,150.3,129.7,126.2,125.4$, 123.9, 122.4, 29.3, 23.9, 15.6; EI-MS 162 [M+', 90\%], 134 [95], 120 [40], 105 [(M20], 91 [100]. The other [Rf=0.6 in EtOAc:hexane (1:4)] was recrystallized from $\mathrm{CHCl}_{3} /$ hexane to give white crystals of 3-(3-methyl-4-hydroxyphenyl)propionitrile ( $840 \mathrm{mg}, 60 \%), \mathrm{mp}$ $94{ }^{\circ} \mathrm{C}$. IR $v_{\max }(\mathrm{KBr}) 3450,2258 ;{ }^{1} \mathrm{H}$ NMR $\left(\mathrm{CDCl}_{3}\right) \delta 6.94(\mathrm{~d}, 2 \mathrm{~Hz}$, $1 \mathrm{H}), 6.90(\mathrm{dd}, 2,8 \mathrm{~Hz}, 1 \mathrm{H}), 6.69(\mathrm{~d}, 8 \mathrm{~Hz}, 1 \mathrm{H}), 5.61(\mathrm{~s}, 1 \mathrm{H}), 2.82(\mathrm{t}$, $7 \mathrm{~Hz}, 2 \mathrm{H}), 2.55(\mathrm{t}, 7 \mathrm{~Hz}, 2 \mathrm{H}), 2.20(\mathrm{~s}, 3 \mathrm{H}) ;{ }^{13} \mathrm{C} \mathrm{NMR}\left(\mathrm{CDCl}_{3}\right) 153.3$, 130.9, 129.8, 126.7, 124.5, 119.5, 115.2, 30.6, 19.7, 15.8; EI-MS 161 [ $\left.\mathrm{M}^{+}, 20 \%\right], 121$ [100], 91 [18], 77 [20].

\subsubsection{Synthesis of}

3-(5-bromo-2-hydroxy-3-methylphenyl)propanoic acid lactone (9h)

Method 1: A solution of $\mathbf{9 i}(1.012 \mathrm{~g}, 5.35 \mathrm{mmol})$ and acrylonitrile $(0.35 \mathrm{~mL}, 0.282 \mathrm{~g}, 5.35 \mathrm{mmol})$ in DCB $(5 \mathrm{~mL})$ was added dropwise to anhyd. $\mathrm{AlBr}_{3}(2.701 \mathrm{~g}, 10.7 \mathrm{mmol})$ suspended in DCB $(20 \mathrm{~mL})$. The mixture was heated at $110^{\circ} \mathrm{C}$ as dry $\mathrm{HCl}$ gas was passed for $5 \mathrm{~h}$, then heated for a further $43 \mathrm{~h}$ at $110^{\circ} \mathrm{C}$, cooled and added to water $(100 \mathrm{~mL})$. The mixture was extracted with $\mathrm{CHCl}_{3}(70 \mathrm{~mL} \times 3)$ and the combined extracts were washed with sat. $\mathrm{NaCl}$ aq., dried $\left(\mathrm{Na}_{2} \mathrm{SO}_{4}\right)$ and evaporated to give a yellow solid $(200 \mathrm{mg})$. Recrystallization from $\mathrm{CHCl}_{3} /$ hexane give white crystalline $9 \mathbf{h}(120 \mathrm{mg}, 10 \%$.), mp $93^{\circ} \mathrm{C}$. IR $v_{\max }(\mathrm{KBr}), 1772 ;{ }^{1} \mathrm{H}$ NMR $\left(\mathrm{CDCl}_{3}\right) \delta 7.24(\mathrm{~d}, 2.5 \mathrm{~Hz}, 1 \mathrm{H})$, $7.16(\mathrm{~d}, 2.5 \mathrm{~Hz}, 1 \mathrm{H}), 2.96(\mathrm{t}, 7 \mathrm{~Hz}, 2 \mathrm{H}), 2.76(\mathrm{t}, 7 \mathrm{~Hz}, 2 \mathrm{H}), 2.27(\mathrm{~s}, 3 \mathrm{H})$; ${ }^{13} \mathrm{C} \mathrm{NMR}\left(\mathrm{CDCl}_{3}\right) 167.9,149.4,132.4,128.5,128.2,124.4,116.3,28.6$, 23.7, 15.4; EI-MS 242 [ $\left.\mathrm{M}^{+},\left({ }^{81} \mathrm{Br}\right), 100 \%\right], 240\left[\mathrm{M}^{+},\left({ }^{79} \mathrm{Br}\right), 100\right], 214$ [84], 212 [84]; 200 [20], 198[20], 172 [22], 170[22], 162 [15], 133 [35], 91[95].

Method 2: To a solution of $\mathbf{9 g}(100 \mathrm{mg}, 0.617 \mathrm{mmol})$ in DCM $(2 \mathrm{~mL})$ was added dropwise a solution of $\mathrm{Br}_{2}(0.98 \mathrm{mg}, 0.617 \mathrm{mmol})$ in 1,2-dichoroethane $(2 \mathrm{~mL})$ at $0^{\circ} \mathrm{C}$, over $30 \mathrm{~min}$. The mixture was stirred for $2 \mathrm{~h}$ at $0^{\circ} \mathrm{C}$, then at $20^{\circ} \mathrm{C}$ overnight. Evaporation under reduced pressure left a residue $(300 \mathrm{mg}$ ), which was dissolved in $\mathrm{CHCl}_{3}(70 \mathrm{~mL})$. The solution was washed with sat. $\mathrm{NaCl}$ aq. $(40 \mathrm{~mL} \times 3)$, dried $\left(\mathrm{Na}_{2} \mathrm{SO}_{4}\right)$ and evaporated to give a white powder $(200 \mathrm{mg})$. Crystallization from benzene/hexane gave white needles of $\mathbf{9 h}$ (141 mg, 95\%), $\mathrm{mp} 93^{\circ} \mathrm{C}$.

\subsubsection{Synthesis of}

3-(5-bromo-2-hydroxy-3-methylphenyl)propanoic acid (9a)

The procedure was like that for $\mathbf{7 a}$ and gave $\mathbf{9 a}$ as a yellow solid in $75 \%$ yield, mp $100{ }^{\circ} \mathrm{C}$. IR $v_{\max }(\mathrm{KBr}) 3500,1750 ;{ }^{1} \mathrm{H}$ NMR (DMSO-d 6 ) $\delta 12.09(\mathrm{~b}, 1 \mathrm{H}), 8.54(\mathrm{~s}, 1 \mathrm{H}), 7.09(\mathrm{~d}, 2.5 \mathrm{~Hz}, 1 \mathrm{H}), 7.06(\mathrm{~d}, 2.5 \mathrm{~Hz}, 1 \mathrm{H})$, $2.76(\mathrm{t}, 8 \mathrm{~Hz}, 2 \mathrm{H}), 2.46(\mathrm{t}, 8 \mathrm{~Hz}, 2 \mathrm{H}), 2.15(\mathrm{~s}, 3 \mathrm{H}) ;{ }^{13} \mathrm{C} \mathrm{NMR}\left(\right.$ DMSO-d $\left._{6}\right)$ $\delta 174.0,152.3,130.8,130.3,129.6,127.4,110.4,33.6,25.4,16.5$; EIMS $260\left[\mathrm{M}^{+},\left({ }^{81} \mathrm{Br}\right), 11 \%\right], 258\left[\mathrm{M}^{+},\left({ }^{79} \mathrm{Br}\right), 11 \%\right], 242$ [45], 240 [45], 214 [40], 212 [40], 200 [10], 198 [10], 188 [68], 186 [70], 162 [80], 134 [70], 120 [20], 107 [80], 91 [100], 77 [72].

\subsubsection{Synthesis of benzyl}

3-(5-bromo-2-hydroxy-3-methylphenyl)propanoate $(\mathbf{9 b})$

The procedure was like that for $\mathbf{6 b}$. $9 \mathbf{b}$ was a colourless oil, $70 \%$ yield. IR $v_{\max }(\mathrm{KBr}) 3350,1713 ;{ }^{1} \mathrm{H} \mathrm{NMR}\left(\mathrm{CDCl}_{3}\right) \delta 7.33(\mathrm{~m}, 5 \mathrm{H})$, 7.12 (d, $2 \mathrm{~Hz}, 1 \mathrm{H}), 7.04$ (d, $2 \mathrm{~Hz}, 1 \mathrm{H}), 5.12(\mathrm{~s}, 2 \mathrm{H}), 2.84(\mathrm{~m}, 2 \mathrm{H}), 2.75$ $(\mathrm{m}, 2 \mathrm{H}), 2.22(\mathrm{~s}, 3 \mathrm{H}) ;{ }^{13} \mathrm{C}$ NMR $\left(\mathrm{CDCl}_{3}\right) \delta 175.7,151.8,135.1,131.8$, 130.5, 129.3, 128.9, 128.5, 128.33, 128.32, 112.1, 67.3, 35.2, 24.4, 16.3;
CI-MS $368\left[\left(\mathrm{M}+\mathrm{NH}_{4}\right)^{+},\left({ }^{81} \mathrm{Br}\right), 30 \%\right], 366\left[\left(\mathrm{M}+\mathrm{NH}_{4}\right)^{+},\left({ }^{79} \mathrm{Br}\right), 30\right], 351$ $\left[(\mathrm{M}+1)^{+}, 15\right], 349\left[(\mathrm{M}+1)^{+}, 15\right], 288$ [5], 261 [29], 180 [100].

\subsubsection{Synthesis of}

4-bromo-6-methyl-2-(2-benzyloxycarbonylethyl)phenyl

acridine9-carboxylate $(\mathbf{9 c})$

The procedure was like that for $\mathbf{6 c}$. 9c was a yellow crystalline solid, $75 \%$ yield, mp $105-106{ }^{\circ} \mathrm{C}$. IR $v_{\max }(\mathrm{KBr}), 1744 ;{ }^{1} \mathrm{H}$ NMR $\left(\mathrm{CDCl}_{3}\right) 8.37$ (d, $\left.9 \mathrm{~Hz}, 2 \mathrm{H}\right), 8.32(\mathrm{~d}, 8 \mathrm{~Hz}, 2 \mathrm{H}), 7.82(\mathrm{~m}, 2 \mathrm{H}), 7.60(\mathrm{~m}$, 2H), 7.4-7.2 (m, 7H), $5.05(\mathrm{~s}, 2 \mathrm{H}), 3.09(\mathrm{t}, 7.5 \mathrm{~Hz}, 2 \mathrm{H}), 2.71(\mathrm{t}, 7.5 \mathrm{~Hz}$, $2 \mathrm{H}), 2.40(\mathrm{~s}, 3 \mathrm{H}) ;{ }^{13} \mathrm{C} \mathrm{NMR}\left(\mathrm{CDCl}_{3}\right) 171.8,165.3,148.7,147.1,135.6$, 134.8, 134.7, 132.9, 132.7, 130.7, 130.4, 130.3, 128.9, 128.5, 128.3, $128.2,127.8,122.8,120.0,66.5,34.1,26.1,18.0$; CI-MS $556\left[(\mathrm{M}+\mathrm{H})^{+}\right.$ $\left.\left({ }^{81} \mathrm{Br}\right) 100 \%\right], 554\left[(\mathrm{M}+\mathrm{H})+\left({ }^{79} \mathrm{Br}\right), 100\right], 476$ [10], 270 [2], 206 [50], 108 [2], 91 [5].

\subsubsection{Synthesis of 4-bromo-6-methyl-2-(2-carboxyethyl)phenyl acridine-9-carboxylate $(\mathbf{9 d})$}

The preparation was like that for $\mathbf{6 d}$. 9c was a yellow crystalline solid, $85 \%$ yield, $\mathrm{mp} 179-180^{\circ} \mathrm{C}$. IR $v_{\max }(\mathrm{KBr}) 3500,1736,1715$; The NMR spectra were more complex than expected, possibly due to mixtures of forms in solution, but the major observed resonances are given; ${ }^{1} \mathrm{H}$ NMR (DMSO- $\left.\mathrm{d}_{6}\right) \delta 12.28(\mathrm{~s}, 1 \mathrm{H}), 8.3-8.4(\mathrm{~m}, 4 \mathrm{H}), 7.96$ (m, 2H), $7.80(\mathrm{~m}, 2 \mathrm{H}), 7.54(\mathrm{~s}, 1 \mathrm{H}), 7.29(\mathrm{~m}, 1 \mathrm{H}), 3.02(\mathrm{~m}, 2 \mathrm{H}), 2.62$ $(\mathrm{m}, 2 \mathrm{H}), 2.40(\mathrm{~s}, 3 \mathrm{H}) ;{ }^{13} \mathrm{C}$ NMR (DMSO-d $\left.\mathrm{d}_{6}\right) 173.1,164.7,148.1,146.7$, 135.4, 134.1, 132.8, 132.0, 130.7, 129.9, 128.1, 126.8, 124.5, 121.8, 119.2, 33.4, 25.4, 17.0; CI-MS $466\left[(\mathrm{M}+\mathrm{H})^{+}\left({ }^{81} \mathrm{Br}\right), 30 \%\right], 464\left[(\mathrm{M}+\mathrm{H})^{+}\right.$ $\left.\left({ }^{79} \mathrm{Br}\right), 30\right], 386$ [100], 224 [5], 206 [10], 180 [60].

\subsubsection{Synthesis of}

4-bromo-6-methyl-2-(2-succinimidyloxycarbonylethyl)phenyl acridine-9-carboxylate $(\mathbf{9 e})$

Compound 9e, 70\% yield, a yellow gum, was prepared like $6 \mathbf{e}$. IR $v_{\max }(\mathrm{KBr}) 1787,1737,1669 ;{ }^{1} \mathrm{H}$ NMR $\left(\mathrm{CDCl}_{3}\right) \delta 8.3-8.4(\mathrm{~m}, 4 \mathrm{H})$, $7.84(\mathrm{~m}, 2 \mathrm{H}), 7.68(\mathrm{~m}, 2 \mathrm{H}), 7.45$ (br s, 2H), 3.17 (t, $7.5 \mathrm{~Hz}, 2 \mathrm{H}), 2.93$ $(\mathrm{t}, 7.5 \mathrm{~Hz}, 2 \mathrm{H}), 2.75(\mathrm{~s}, 4 \mathrm{H}), 2.44(\mathrm{~s}, 3 \mathrm{H}) ;{ }^{13} \mathrm{C} \mathrm{NMR}\left(\mathrm{CDCl}_{3}\right) 168.8$, 167.4, 165.3, 148.7, 147.1, 134.5, 133.5, 133.2, 133.1, 130.8, 130.43, 130.36, 128.0, 124.7, 122.7, 120.2, 31.0, 25.7, 25.5, 17.8; FAB-MS 585 $\left.\left[(\mathrm{M}+\mathrm{Na})^{+},\left({ }^{81} \mathrm{Br}\right), 10 \%\right], 583[\mathrm{M}+\mathrm{Na})^{+},\left({ }^{79} \mathrm{Br}\right), 10\right], 563\left[(\mathrm{M}+\mathrm{H})^{+}, 54\right]$, $561\left[(\mathrm{M}+\mathrm{H})^{+}, 54\right], 225$ [96], 206 [100], 179 [64].

\subsubsection{Synthesis of}

4-bromo-6-methyl-2-(2-succinimidyloxycarbonylethyl)phenyl 10 methylacridinium-9-carboxylate trifluoromethanesulfonate (9)

A procedure as for 6 gave yellow solid 9, 82\%, mp 190-192 ${ }^{\circ} \mathrm{C}$. IR $v_{\max }(\mathrm{KBr}) 1800,1760,1750$; NMR (DMSO-d6) ${ }^{1} \mathrm{H} \delta 8.95$ (d, $9 \mathrm{~Hz}, 2 \mathrm{H}$ ), $8.55(\mathrm{~d}, 9 \mathrm{~Hz}, 2 \mathrm{H}), 8.35(\mathrm{~m}, 2 \mathrm{H}), 8.15(\mathrm{~m}, 2 \mathrm{H}), 7.57(\mathrm{~s}, 1 \mathrm{H}), 7.56(\mathrm{~s}, 1 \mathrm{H})$, $4.97(\mathrm{~s}, 3 \mathrm{H}), 2.97(\mathrm{~m}, 2 \mathrm{H}), 2.63(\mathrm{~m}, 2 \mathrm{H}), 2.59(\mathrm{~s}, 4 \mathrm{H}), 2.50(\mathrm{~s}, 3 \mathrm{H}) ;{ }^{13} \mathrm{C}$ $\delta$ 173.7, 164.0, 162.6, 141.9, 145.3, 146.7, 139.2, 136.7, 135.5, 133.3, 132.2, 131.4, 130.5, 129.8, 127.0, 122.3, 120.1, 40.1, 33.3, 29.5, 25.2, 17.5; FAB-MS $577\left[\left(\mathrm{M}-\mathrm{SO}_{3} \mathrm{CF}_{3}\right)^{+},\left({ }^{81} \mathrm{Br}\right), 5 \%\right], 575\left[\left(\mathrm{M}-\mathrm{SO}_{3} \mathrm{CF}_{3}\right)^{+}\right.$, $\left.\left({ }^{79} \mathrm{Br}\right), 5\right], 562$ [10], 480 [100], 464 [34], 221 [58], 207 [7], 193[35], 179 [61].

\subsubsection{Synthesis of benzyl 3-(3-hydroxyphenyl)propanoate (10b)}

A mixture of 10a $(0.780 \mathrm{~g}, 4.70 \mathrm{mmol})$ in $\mathrm{MeOH}(5 \mathrm{~mL})$ and $\mathrm{KOH}(0.268 \mathrm{~g}, 4.70 \mathrm{mmol})$ in $\mathrm{MeOH}(25 \mathrm{~mL})$ was stirred at $20^{\circ} \mathrm{C}$ for $1 \mathrm{~h}$. The solvent was evaporated and the residue dried under vacuum. This $\mathrm{K}^{+}$salt $(0.800 \mathrm{~g}, 3.90 \mathrm{mmol})$ and dibenzo-18-crown-6 $(0.143 \mathrm{~g}, 0.44 \mathrm{mmol})$ were stirred at $80-90^{\circ} \mathrm{C}$ for $30 \mathrm{~min}$ in DMFacetonitrile $(16 \mathrm{~mL}, 1: 2, \mathrm{v} / \mathrm{v})$, benzyl chloride $(0.63 \mathrm{~mL}, 4.4 \mathrm{mmol})$ was added and stirring maintained for $5 \mathrm{~h}$. The solid formed was filtered off and the filtrate evaporated to give a thick brown liquid. The crude product was purified by column chromatography (silica, $\mathrm{CHCl}_{3}$ ) and fractions with a component having $\mathrm{Rf}=0.63$ (silica 
TLC, toluene-ethyl acetate, 4:1) were collected. Evaporation of solvent gave 10b $(0.844 \mathrm{~g}, 84 \%)$ as a thick yellow liquid. IR $v_{\max }$ (thin film). 3385, 1732; ${ }^{1} \mathrm{H} \mathrm{NMR}\left(\mathrm{CDCl}_{3}\right)$ 7.27-7.40 (m, 5H), 7.14 (apparent $\mathrm{t}, 8 \mathrm{~Hz}, 1 \mathrm{H}), 6.68-6.76(\mathrm{~m}, 3 \mathrm{H}), 5.86(\mathrm{~b}, 1 \mathrm{H}), 5.14(\mathrm{~s}, 2 \mathrm{H}), 2.93(\mathrm{t}$, $7.5 \mathrm{~Hz}, 2 \mathrm{H}), 2.71(\mathrm{t}, 7.5 \mathrm{~Hz}, 2 \mathrm{H}) ;{ }^{13} \mathrm{CNMR}\left(\mathrm{CDCl}_{3}\right) \delta 173.2,155.9,142.1$, 135.8, 129.4, 128.7, 128.7, 128.3, 120.5, 115.3, 113.4, 66.6, 35.8, 30.8 FAB-MS $m / z 279$ [(M+Na) $\left.)^{+}, 26 \%\right], 256$ [ $\left.\mathrm{M}^{+}, 99\right], 165$ [65], 107 [100]; Anal. Calcd. for $\mathrm{C}_{16} \mathrm{H}_{16} \mathrm{O}_{3}$ : C, 74.98\%; H, 6.29\%; Found: C, 74.66\%; $\mathrm{H}$, $6.18 \%$.

\subsubsection{Synthesis of 3-(2-benzyloxycarbonylethyl)phenyl} acridine-9-carboxylate (10c)

A mixture of $10 \mathbf{b}(0.613 \mathrm{~g}, 2.40 \mathrm{mmol})$ and acridine-9-carboxyl chloride $(0.610 \mathrm{~g}, 2.52 \mathrm{mmol})$ in anhyd. pyridine (previously stored over $3 \mathrm{~A}$ molecular sieves, $10 \mathrm{~mL}$ ) was stirred at $20^{\circ} \mathrm{C}$ overnight then poured into hydrochloric acid $(50 \mathrm{~mL}, 1 \mathrm{M}) .3 \mathrm{c}(0.680 \mathrm{~g}, 56 \%)$, was collected as a pale yellow solid by filtration, $\mathrm{mp} 106-108^{\circ} \mathrm{C}$. This was used in the subsequent synthesis, but a sample was purified by column chromatography (silica, $\mathrm{CHCl}_{3}$-ethyl acetate $4: 1$ ). Fractions having $\mathrm{Rf}=0.64$ (silica, toluene-ethyl acetate, $4: 1$ ) were combined . Evaporation gave pale yellow solid 3c. IR $v_{\max }(\mathrm{KBr}), 1755,1722 ;{ }^{1} \mathrm{H}$ $\operatorname{NMR}\left(\mathrm{CDCl}_{3}\right) \delta 8.31(\mathrm{~d}, 9 \mathrm{~Hz}, 2 \mathrm{H}), 8.21(\mathrm{~d}, 8.5 \mathrm{~Hz}, 2 \mathrm{H}), 7.83$ (dd, 8.5, $7 \mathrm{~Hz}, 2 \mathrm{H}$ ), 7.66 (dd, 9, $7 \mathrm{~Hz}, 2 \mathrm{H}$ ), 7.43 (apparent t, $8 \mathrm{~Hz}, 1 \mathrm{H}$ ), 7.24-7.35 $(\mathrm{m}, 7 \mathrm{H}), 7.15(\mathrm{~d}, 8 \mathrm{~Hz}, 1 \mathrm{H}), 5.14(\mathrm{~s}, 2 \mathrm{H}), 3.08(\mathrm{t}, 8 \mathrm{~Hz}, 2 \mathrm{H}), 2.77(\mathrm{t}, 8 \mathrm{~Hz}$, $2 \mathrm{H}) ;{ }^{13} \mathrm{CNMR}\left(\mathrm{CDCl}_{3}\right) \delta 172.6,165.9,150.5,148.6,142.6,135.8,135.8$, $130.5,130.0,129.9,128.6,128.5,128.3,127.5,126.7,124.9,122.4$, $121.4,119.4,66.5,35.7,30.8$; FAB-MS $m / z 484$ [(M+Na $\left.)^{+}, 11 \%\right], 462$ [(M+H $\left.)^{+}, 100\right], 206$ [49]; Anal. Calcd.for $\mathrm{C}_{30} \mathrm{H}_{23} \mathrm{NO}_{4}$ : C, 78.08\%; $\mathrm{H}$, $5.02 \%, \mathrm{~N}, 3.03 \%$; Found: C, $77.90 \%$; H, 5.11\%, N, 2.99\%.

\subsubsection{Synthesis of 3-(2-carboxyethyl)phenyl} acridine-9-carboxylate (10d)

The procedure was like that for $\mathbf{6 d}$, giving $10 d$ in $78 \%$ yield, $\mathrm{mp}$ 194-195 ${ }^{\circ} \mathrm{C}$. IR $v_{\max }(\mathrm{KBr}) 3056,1749,1729 ;{ }^{1} \mathrm{H}$ NMR (DMSO-d 6 ) $8.31(\mathrm{~d}, 9 \mathrm{~Hz}, 2 \mathrm{H}), 8.30(\mathrm{~d}, 9 \mathrm{~Hz}, 2 \mathrm{H}), 8.01(\mathrm{~m}, 2 \mathrm{H}), 7.84(\mathrm{~m}, 2 \mathrm{H})$, 7.44-7.52 (m, 3H), $7.30(\mathrm{~d}, 7 \mathrm{~Hz}, 1 \mathrm{H}), 2.98(\mathrm{t}, 8 \mathrm{~Hz}, 2 \mathrm{H}), 2.66(\mathrm{t}, 8 \mathrm{~Hz}$, $1 \mathrm{H})$ (the exchangeable proton was not observed); ${ }^{13} \mathrm{C}$ NMR (DMSO$\left.\mathrm{d}_{6}\right) \delta 173.6,165.5,150.1,147.9,143.4,135.6,131.0,129.7,129.5$, $128.1,126.7,124.9,121.7,121.5,119.5,35.1,30.2 ;$ FAB-MS $m / z 394$ $\left[(\mathrm{M}+\mathrm{Na})^{+}, 12 \%\right], 372\left[(\mathrm{M}+\mathrm{H})^{+}, 100\right], 206$ [46], 179 [54]; Anal. Calcd. for $\mathrm{C}_{23} \mathrm{H}_{17} \mathrm{NO}_{4}$ : C, 74.38\%; $\mathrm{H}, 4.61 \%$; N, 3.77\%; Found: $\mathrm{C}, 74.58 \% ; \mathrm{H}$, $4.39 \%$;, $3.98 \%$.

\subsubsection{Synthesis of 3-(2-succinimidyloxycarbonylethyl)phenyl acridine-9-carboxylate (10e)}

10e was prepared like 4e. Yield 67\%; mp 202-203 ${ }^{\circ} \mathrm{C}$. IR $v_{\max }$ $(\mathrm{KBr}), 1818,1784,1753,1734 ;{ }^{1} \mathrm{H} \mathrm{NMR}\left(\mathrm{CDCl}_{3}\right) \delta 8.31(\mathrm{~d}, 9 \mathrm{~Hz}, 2 \mathrm{H})$, $8.25(\mathrm{~d}, 9 \mathrm{~Hz}, 2 \mathrm{H}), 7.85(\mathrm{dd}, 9,7 \mathrm{~Hz}, 2 \mathrm{H}), 7.68(\mathrm{dd}, 9,7 \mathrm{~Hz}, 2 \mathrm{H}), 7.49$ $(\mathrm{m}, 1 \mathrm{H}), 7.33-7.35(\mathrm{~m}, 2 \mathrm{H}), 7.26(\mathrm{~s}, 1 \mathrm{H}), 3.18(\mathrm{t}, 8 \mathrm{~Hz}, 2 \mathrm{H}), 3.00(\mathrm{t}$, $8 \mathrm{~Hz}, 2 \mathrm{H}), 2.83(\mathrm{~s}, 4 \mathrm{H}) ;{ }^{13} \mathrm{C} \mathrm{NMR}\left(\mathrm{CDCl}_{3}\right) \delta 169.0,167.7,165.9,150.7$, 148.7, 141.3, 135.8, 130.4, 130.2, 130.1, 127.6, 126.6, 125.0, 122.4, $121.5,119.9,32.5,30.3,25.6$; FAB-MS $m / z 491$ [(M+Na $\left.)^{+}, 11 \%\right], 469$ $\left[(\mathrm{M}+\mathrm{H})^{+}, 100\right], 206$ [17], 179 [40]; Anal. Calcd. for $\mathrm{C}_{27} \mathrm{H}_{20} \mathrm{~N}_{2} \mathrm{O}_{6}$ : C, 69.23\%; H, 4.30\%; N, 5.98\%; Found: C, 69.04\%; H, 4.08\%; N, 5.87\%.

\subsubsection{Synthesis of 3-(2-succinimidyloxycarbonylethyl)phenyl 10-methylacridinium-9-carboxylate trifluoromethanesulfonate (10)}

The procedure was like that for $\mathbf{6}$, giving $\mathbf{1 0}$ as a yellow solid, $71 \%$ yield, mp $158-161{ }^{\circ} \mathrm{C}$. IR $v_{\max }(\mathrm{KBr}), 1813,1785,1757,1738$; ${ }^{1} \mathrm{H}$ NMR (DMSO-d $) \delta 8.96(\mathrm{~d}, 9 \mathrm{~Hz}, 2 \mathrm{H}), 8.64(\mathrm{~d}, 9 \mathrm{~Hz}, 2 \mathrm{H}), 8.57(\mathrm{~m}$, $2 \mathrm{H},), 8.24(\mathrm{~m}, 2 \mathrm{H}), 7.71(\mathrm{~s}, 1 \mathrm{H}), 7.54-7.64(\mathrm{~m}, 2 \mathrm{H}), 7.44(\mathrm{~d}, 7.5 \mathrm{~Hz}, 1 \mathrm{H})$, $4.97(\mathrm{~s}, 3 \mathrm{H}), 3.10-3.19(\mathrm{~m}, 4 \mathrm{H}), 2.84(\mathrm{~s}, 4 \mathrm{H}) ;{ }^{13} \mathrm{C}$ NMR $\left(\right.$ DMSO- $\left._{6}\right) \delta$ 170.2, 168.4, 163.6, 149.6, 146.9, 142.1, 142.0, 139.3, 130.1, 129.8, $127.7,127.7,127.6,122.3,121.7,120.0,39.9,31.5,29.6,25.5\left(\mathrm{CF}_{3} \mathrm{car}-\right.$ bon not observed); FAB-MS $m / z 483$ [(M- $\left.\mathrm{SO}_{3} \mathrm{CF}_{3}\right)^{+}, 99 \%$ ], 221 [35],
193 [100]; Anal. Calcd. for $\mathrm{C}_{29} \mathrm{H}_{23} \mathrm{~N}_{2} \mathrm{~F}_{3} \mathrm{O}_{9} \mathrm{~S}$ : C, 55.06\%; H, 3.66\%; N, 4.43\%; Found: C, 55.03\%; H, 3.71\%, N, 4.34\%.

\subsection{Chemiluminescent kinetics and efficiency}

A standard procedure was employed for estimation of chemiluminescent kinetics and efficiency. The samples (compounds $\mathbf{1}$ and 6-10, around $1 \mathrm{mg}$ each) were separately dissolved in anhydrous acetonitrile $(1.0 \mathrm{~mL})$. The solutions were diluted to $1 \times 10^{-10} \mathrm{~mol} / \mathrm{L}$ using $1.00 \times 10^{-3} \mathrm{M}$ hydrochloric acid. To a test tube containing the diluted sample solution $(10 \mu \mathrm{L})$, hydrogen peroxide solution $\left(0.3 \mathrm{~mL}\right.$ of $0.5 \%, \mathrm{w} / \mathrm{v}$ in $\left.0.1 \mathrm{~mol} / \mathrm{L} \mathrm{HNO}_{3}\right)$ was delivered automatically, followed by $\mathrm{NaOH}$ solution $(0.3 \mathrm{~mL}$ of $0.25 \mathrm{~mol} / \mathrm{L})$ containing a surfactant. The output of photons was counted for $3 \mathrm{~s}$ (compounds $\mathbf{1}, \mathbf{8}, \mathbf{1 0}$ ), 10 s (compound 6), or $200 \mathrm{~s}$ (compounds 7, 9). Chemiluminescent intensity versus time curves were plotted, from which chemiluminescent half peak times and life times were measured. The total counts were used to calculate the relative chemiluminescent efficiencies for all compounds.

\section{Results and discussion}

\subsection{Syntheses of compounds $\mathbf{6}, \mathbf{7}, \mathbf{8}, \mathbf{9}$ and $\mathbf{1 0}$}

Scheme 2 outlines the syntheses of the acridinium ester labels from the appropriate 3-(2-hydroxyphenyl)propanoic acid derivatives 6a-9a or 3-(3-hydroxyphenyl)propanoic acid, 10a. Firstly, the carboxylic acid groups were protected by benzylation. The benzyl esters 6b-10b were then coupled to acridine-9-carbonyl chloride, which was prepared by reacting acridine-9-carboxylic acid with thionyl chloride. [7] Deprotection of $\mathbf{6 c - 1 0 c}$ with a mixture of ethanoic acid and $\mathrm{HBr}$ yielded acids $\mathbf{6 d - 1 0 d}$, which were then esterified with $\mathrm{N}$-hydroxysuccinimide in the presence of dicyclohexylcarbodiimide (DCC) to give 6e-10e. Finally, methylation by treatment with methyl trifluoromethanesulfonate under an atmosphere of nitrogen afforded the target molecules 6-10. All of the reactions gave good yields (given in parentheses after the structure number for the compounds synthesized as recorded in Scheme 2).

Compound 10a was commercially available. The syntheses of the substituted 3-(2-hydroxyphenyl)propanoic acids 6a-9a are summarised in Scheme 3. Figures in parentheses under the reaction arrows are the yields obtained.

3-(2-Hydroxyphenyl)propanoic acid (6a) was prepared by hydrogenation of 3-(2-hydroxyprop-2-enoic acid (6f) over a palladium on carbon catalyst. The synthesis of 3-(2-hydroxy-3,5-dimethylphenyl)propanoic acid (7a) was begun with conversion of 2,4dimethylphenol (7f) to 3-(2-hydroxy-3,5-dimethylphenyl)propanoic acid lactone $(\mathbf{7 g})$ by reaction with acrylonitrile under catalysis by $\mathrm{AlCl}_{3}$ and $\mathrm{HCl}$. The resulting lactone was subsequently hydrolysed in base and the salt formed was converted to 7a by acidification. 3-(3,5-Dibromo-2-hydroxyphenyl)propanoic acid (8a) was obtained by direct bromination of 3-(2-hydroxyphenyl)propanoic acid $(\mathbf{6 a})$.

The synthesis of 3-(5-bromo-2-hydroxy-3-methyl-phenyl)propanoic acid (9a) was carried out by initial selective monobromination of 0 -cresol (9f) to 4-bromo-2 methylphenol (9i), subsequent formation of the lactone $(\mathbf{9 b})$ by reaction with acrylonitrile under catalysis by $\mathrm{AlCl}_{3}$ and $\mathrm{HCl}$, and finally by hydrolysis of the lactone to 9a. However, the step of making the lactone $(\mathbf{9 b})$ suffered from a low yield (10\%). No improvement in yield was achieved even after variation of the solvent, catalyst, temperature and reaction time. The reason for the low yield appears to be that the electrophilic substitution reaction is deactivated by the electron withdrawing nature of the $\mathrm{Br}$ group. An alternative approach was tried. In this approach, the Friedel-Crafts substitution and subsequent cyclisation to form the lactone was carried out prior to the bromination step, thereby 


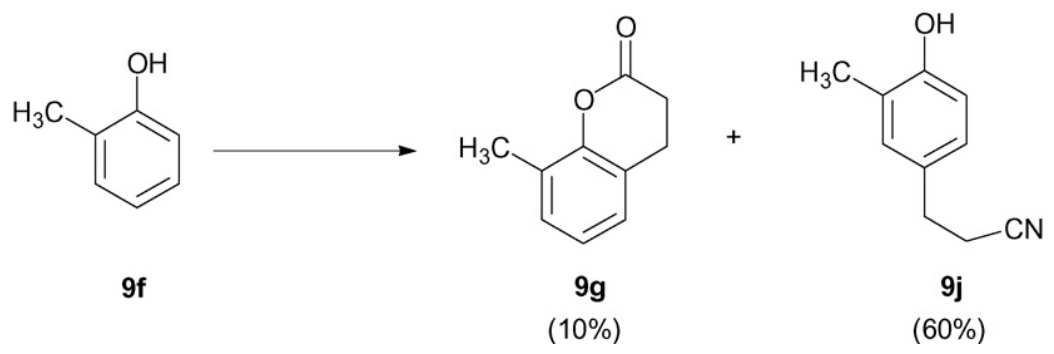

Scheme 4. Selectivity problem in the synthesis of $\mathbf{9 g}$.

avoiding the deactivating effect of the bromo group. However, this approach had a problem of selectivity in the step involving preparation of the lactone. The desired product was obtained in only $10 \%$ yield, while undesired 3-(3-methyl-4-hydroxyphenyl)propionitrile (9j, Scheme 4) was produced by the reaction in a yield of $60 \%$.

Various attempts to improve the yield of $\mathbf{9 g}$ by variation of the reaction conditions failed to give significant improvement. Therefore, it was decided to continue the route with the small amount of material available. The final product $\mathbf{9}$ was produced in sufficient quantity to allow evaluation of its chemiluminescent properties.

\subsection{Chemiluminescent properties}

\subsubsection{Chemiluminescence kinetics}

The emission of light on oxidation of each of the compounds 1 and $\mathbf{6}$ through $\mathbf{1 0}$ was studied over time under a particular set of conditions (see Section 2) and the period of time for which luminescence could be detected (chemiluminescent lifetime) was determined. The results are recorded in Table 1 , which illustrates that the chemiluminescent lifetimes are in the order $7>9>6>10 \bumpeq 1>8$.

The rate-determining step of the chemiluminescent reaction of acridinium esters involves cleavage of the phenoxy moiety. [8] Electron withdrawing substituents like $\mathrm{Br}$ would lead to the phenoxy moiety becoming a better leaving group, whereas electron donating groups like $\mathrm{CH}_{3}$ would cause the phenoxy moiety to become a relatively poor leaving group. Therefore, on electronic grounds the order of reactivity would be expected to be like the observed order. In addition, the results shown in Table 1 suggest that the effects of steric hindrance could also play a role in determining the kinetics of the chemiluminescence reaction because the more sterically hindered $\mathbf{6}$ showed significantly slower reaction kinetics that its less sterically hindered isomers $\mathbf{1}$ and $\mathbf{1 0}$.

Table 1

Chemiluminescent lifetime for the new compounds.

\begin{tabular}{lc}
\hline Compound & Lifetime $(\mathrm{sec})$ \\
\hline $\mathbf{1}$ & 3 \\
$\mathbf{6}$ & 10 \\
$\mathbf{7}$ & 180 \\
$\mathbf{8}$ & 2 \\
$\mathbf{9}$ & 122 \\
$\mathbf{1 0}$ & 3 \\
\hline
\end{tabular}

Table 2

Relative chemiluminescent efficiencies of the new compounds.

\begin{tabular}{lc}
\hline Compound & Relative chemiluminescent efficiency \\
\hline 1 & 100 \\
6 & 166 \\
7 & 252 \\
8 & 45 \\
9 & 428 \\
10 & 85 \\
\hline
\end{tabular}

\subsubsection{Chemiluminescent efficiency}

Chemiluminescent efficiency of acridinium esters is generally affected by structural modifications, but the results are not always predictable. The relative chemiluminescent efficiencies of $\mathbf{6 , 7 , 8 , 9}$ and $\mathbf{1 0}$ have therefore been examined in comparison with that of $\mathbf{1}$, and the results are summarised in Table 2. As the results show, compounds 6, 7, and 9 show a much higher chemiluminescent efficiency than compound $\mathbf{1}$, especially compound $\mathbf{9}$, which has over four-fold higher chemiluminescence efficiency than compound $\mathbf{1}$. Compounds 8 and $\mathbf{1 0}$ are less efficient, with compound $\mathbf{8}$ having the lowest efficiency, half of that of compound $\mathbf{1}$.

\section{Conclusions}

Several ortho- and meta-linked phenyl acridinium ester labelling compounds, differing in the substituents on the phenyl group, have been successfully prepared in reasonable yields. All exhibit chemiluminescence when reacted with hydrogen peroxide and the ortho-linked compounds except for the dibromo derivative show significantly improved quantum yields of luminescence. The 4-bromo-6-methyl derivative exhibits a quantum yield of luminescence that is over four-fold higher than the unsubstituted para-linked analogue, which is widely used in biological assays. Both steric and particularly electronic effects are important in determining the rate at which the chemiluminescent reaction occurs, and this has thereby provided a range of labels which are differentiated in the time over which light is emitted. This provides opportunities for choosing a label with properties appropriate for a particular application. For example, the dibromo compound flashes quickly while the 4,6-dimethyl and 4-bromo-6-methyl compounds glow slowly, as a result of the relative ease of expulsion of the corresponding phenoxide anions.

\section{Acknowledgements}

This work was funded by Molecular Light Technology Research Ltd., Cardiff, United Kingdom. We thank the EPSRC and the University of Wales for grants that enabled the purchase of NMR equipment used in this study and the EPSRC National Mass Spectrometry Service Centre in Swansea for running the mass spectra.

\section{References}

[1] I. Weeks, I. Beheshti, F. McCapra, A.K. Campbell, J.S. Woodhead, Clin. Chem. 29 (1983) 1474

[2] N.C. Nelson, P.W. Hammond, E. Matsuda, A.A. Goud, M.M. Becker, Nucleic Acids Res. 24 (1996) 4998.

[3] K. Smith, Z. Li, J.-J. Yang, I. Weeks, J.S. Woodhead, J. Photochem. Photobiol. A: Chem. 132 (2000) 181.

[4] E. Pretsch, T. Clerc, J. Seibl, W. Simon, Strukturaufklarung Organischer Verbindungen, Springer-Verlag, Berlin, 1976.

[5] K.L. Amsberry, R.T. Borchardt, J. Org. Chem. 55 (1990) 5867.

[6] E. Klarmann, L.W. Gates, V.A. Shternov, P.H. Cox, J. Am. Chem. Soc. 55 (1933) 4657.

[7] (a) M.M. Rauhut, D. Sheehan, R.A. Clarke, B.G. Roberts, A.M. Semsel, J. Org. Chem. 30 (1965) 3587;

(b) E. Rapaport, M.W. Cass, E.H. White, J. Am. Chem. Soc. 94 (1972) 3153.

[8] F. McCapra, D. Watmore, F. Sumun, A. Patel, I. Behesti, K. Ramakrishnan, J. Branson, J. Biolumin. Chemilumin. 4 (1989) 51. 